\title{
Shaped Modal Sensors for Linear Stochastic Beams
}

\author{
S. ADHIKARI* AND M. I. FRISWELL \\ School of Engineering, Swansea University, Swansea, SA2 8PP, UK
}

\begin{abstract}
Modal sensors and actuators using distributed piezoelectric material have a wide range of applications, for example in vibration control and piezoelectric transformers. The design of these transducers usually ignores any uncertainty and variability in the host structure, which can have a significant effect on their performance. This article investigates the design of shaped piezoelectric sensors for beam structures that are robust with respect to uncertainties in the system. The modal transducers are defined using a discrete approximation to the equations of motion for linear stochastic systems and their shapes are represented using the underlying finite element shape functions. The optimal shape design has been coupled with the stochastic finite element method to consider parametric uncertainty described using random fields, using a first-order perturbation-based approach to obtain the second-order covariance of the modal matrix. The numerical results for linear elastic beam structures showed that the shape of the sensors of the stochastic system can differ significantly from the corresponding deterministic system. However, sensors with shapes designed using a smoothness criterion also perform very well for structures with uncertainty.
\end{abstract}

Key Words: sensor, optimization, structural health monitoring, uncertainty, random fields, stochastic finite element.

\section{INTRODUCTION}

$\mathbf{T}$ HE idea of using modal sensors and actuators for beam and plate type structures has been a subject of intense interest for many years. Using modal sensors in active control reduces problems of spillover, where high frequency unmodeled modes affect the stability of the closed-loop system. This article is concerned with distributed sensors made of piezoelectric material to measure the response of beam and plate structures. The design of modal sensors for beam structures is well established, where a sensor may be obtained by varying the sensor width along the length of the beam. If the sensor covers the whole beam the shape of the sensor may be derived using the mode shape orthogonality property (Lee and Moon, 1990). Friswell (1999) considered modal sensors that cover only part of the beam, segmented modal sensors for multiple modes, and the effect of geometric tolerances during manufacture on the quality of the sensors. Friswell (2001) parameterized the width of the sensor for beam structures using the finite element method and used the underlying shape functions to approximate the transducer shape. Elka and Bucher (2009) investigated the design of

*Author to whom correspondence should be addressed. E-mail: S.Adhikari@swansea.ac.uk

Figures 1-3 appear in color online: http://jim.sagepub.com shaped sensors for micro-electro-mechanical structures. For plate structures constant thickness sensors are difficult to design, although methods that parameterize sensor boundary, or using topology optimization, have been suggested (Mukherjee and Joshi, 2002; Jian and Friswell, 2006; Donoso and Bellido, 2009a,b). Currently modal sensors and actuators are designed using deterministic models of the structure, and assuming that they may be manufactured perfectly. The performance of the transducers is critically dependent on these assumptions and this article investigates the effect of uncertainty in the modeling of the structure. Piezoelectric sensors are being increasing used in microelectronic devices (for example in piezoelectric transformers, Huang et al. (2007a,b)), where the uncertainty and variability in the structure is likely to be significant.

The consideration of uncertainties in numerical models to obtain the probabilistic descriptions of vibration response and robustness of vibration control with respect to uncertainties is becoming more desirable for industrial scale finite element models. Such uncertainties include, but are not limited to (a) parametric uncertainty (e.g., uncertainty in geometric parameters, friction coefficient, strength of the materials involved); (b) model uncertainty (arising from the lack of scientific knowledge about the model which is unknown a priori); (c) experimental error (uncertain and unknown errors percolate into the model when they are calibrated against experimental results); and (d) 
computational uncertainty (e.g., machine precision, error tolerance and the so called ' $h$ ' and ' $p$ ' refinements in finite element analysis). These uncertainties must be assessed and managed for robust vibration control and sensor design.

In this article we focus our attention on dynamical systems with parametric uncertainties. In the parametric approach, the uncertainties associated with the distributed system parameters, such as Young's modulus, mass density, Poisson's ratio, and geometric parameters are quantified using statistical methods. Problems of structural dynamics in which the uncertainty in specifying mass, damping, and stiffness of the structure is modeled within the framework of random fields can be treated using the stochastic finite element method, see for example Ghanem and Spanos (1991), Kleiber and Hien (1992), and Manohar and Adhikari (1998a,b). The application of the stochastic finite element method to linear structural dynamic problems typically consists of the following key steps:

(1) Selection of appropriate probabilistic models for parameter uncertainties and boundary conditions

(2) Replacement of the element property random fields by an equivalent set of a finite number of random variables. This step, known as the 'discretization of random fields', is a major step in the analysis.

(3) Formulation of the discretized equation of motion.

Following the above procedure, the stochastic finite element model of the structure can be represented in the form:

$$
\begin{gathered}
\mathbf{M}(\theta) \ddot{\mathbf{q}}+\mathbf{D}(\theta) \dot{\mathbf{q}}+\mathbf{K}(\theta) \mathbf{q}=\mathbf{B u}, \\
\mathbf{y}=\mathbf{C} \mathbf{q} .
\end{gathered}
$$

Here $\mathbf{M}(\theta), \mathbf{D}(\theta)$, and $\mathbf{K}(\theta)$ are the random mass, damping, and stiffness matrices based on the degrees of freedom, $\mathbf{q}$, respectively. The inputs to the structure, u, are applied via a matrix $\mathbf{B}$ which determines the location and gain of the actuators (or the actuator shape for distributed actuators). Similarly the outputs, $\mathbf{y}$, are obtained via the output matrix $\mathbf{C}$ which is determined by the sensor shape. The notation $\theta$ is used to denote the random nature of the system matrices.

This article develops a method to design shaped modal sensors for Euler-Bernoulli beams with uncertain properties. It will be shown that due to the uncertain nature of the system matrices, several mathematical choices are possible for optimal design. Here a simple and physically realistic approach is developed and the resulting formulation is applied to a beam with bending stiffness and mass per unit length modeled as a stationary normal random field with a given mean value and exponentially decaying auto-correlation function.

\section{DEFINING SHAPED SENSORS FOR BEAM STRUCTURES}

The shape of a transducer is a continuous function. However, this function needs to be parameterized to enable the optimization of the sensor shape. Using the shape functions of the underlying finite element model is a convenient approach to approximate the width of the piezoelectric material. In this way modal transducers may be designed for arbitrary beam type structures. Furthermore modal transducers that only cover part of a structure may be designed. Most of the development will concern sensors, although actuators may be dealt with in a similar way.

Suppose a single polyvinylidene fluoride film sensor is placed on the beam with a shape defined by a variable width $f(\xi)$, where $\xi$ denotes the length along the beam element. Incorporated into $f(\xi)$ is both the physical width of the sensor, and also the polarization profile of the material. The central feature of the proposed method is to approximate this width using the shape functions of the underlying finite element model. For an Euler-Bernoulli beam these shape functions, for element number $e$, are:

$$
\begin{aligned}
& N_{e 1}(\xi)=\left(1-3 \frac{\xi^{2}}{\ell_{e}^{2}}+2 \frac{\xi^{3}}{\ell_{e}^{3}}\right), \\
& N_{e 2}(\xi)=\ell_{e}\left(\frac{\xi}{\ell_{e}}-2 \frac{\xi^{2}}{\ell_{e}^{2}}+\frac{\xi^{3}}{\ell_{e}^{3}}\right) \\
& N_{e 3}(\xi)=\left(3 \frac{\xi^{2}}{\ell_{e}^{2}}-2 \frac{\xi^{3}}{\ell_{e}^{3}}\right) \\
& N_{e 4}(\xi)=\ell_{e}\left(-\frac{\xi^{2}}{\ell_{e}^{2}}+\frac{\xi^{3}}{\ell_{e}^{3}}\right)
\end{aligned}
$$

where $\ell_{e}$ is the length of the element. Thus the sensor width within element number $e$ is approximated as:

$$
f_{e}(\xi)=\left[\begin{array}{llll}
N_{e 1}(\xi) & N_{e 2}(\xi) & N_{e 3}(\xi) & N_{e 4}(\xi)
\end{array}\right]\left\{\begin{array}{l}
f_{e 1} \\
f_{e 2} \\
f_{e 3} \\
f_{e 4}
\end{array}\right\}
$$

where the constants $f_{e i}$ must be determined. This approximation has the advantage that the width and slope of the sensor are continuous at the nodes of the finite element model. The output (voltage or charge) from the part of the sensor with element number $e$ is:

$$
y_{e}(t)=K_{s} \int_{0}^{\ell_{e}} f_{e}(\xi) \frac{\partial^{2} w_{e}(\xi, t)}{\partial^{2} \xi} \mathrm{d} \xi,
$$

where the constant $K_{s}$ is determined by the properties of the piezoelectric material and $w_{e}$ is the translational 
displacement of the beam (Lee and Moon, 1990; Hsu et al., 1998). This displacement is also approximated by the shape functions as:

$$
w_{e}(\xi)=\left[\begin{array}{llll}
N_{e 1}(\xi) & N_{e 2}(\xi) & N_{e 3}(\xi) & N_{e 4}(\xi)
\end{array}\right]\left\{\begin{array}{l}
w_{e 1} \\
w_{e 2} \\
w_{e 3} \\
w_{e 4}
\end{array}\right\} .
$$

Combining Equations (4)-(6) gives the sensor output for the element as:

$$
y_{e}=\left\{\begin{array}{l}
f_{e 1} \\
f_{e 2} \\
f_{e 3} \\
f_{e 4}
\end{array}\right\}^{\top} \mathbf{C}_{e}\left\{\begin{array}{l}
w_{e 1} \\
w_{e 2} \\
w_{e 3} \\
w_{e 4}
\end{array}\right\}
$$

where the $(i, j)$ th element of the matrix $\mathbf{C}_{e}$ is:

$$
\mathbf{C}_{e i j}=K_{s} \int_{0}^{\ell_{e}} N_{e i}(\xi) N_{e j}^{\prime \prime}(\xi) \mathrm{d} \xi
$$

giving

$$
\mathbf{C}_{e}=-\frac{K_{s}}{30 \ell_{e}}\left[\begin{array}{cccc}
36 & 33 \ell_{e} & -36 & 3 \ell_{e} \\
3 \ell_{e} & 4 \ell_{e}^{2} & -3 \ell_{e} & -\ell_{e}^{2} \\
-36 & -3 \ell_{e} & 36 & -33 \ell_{e} \\
3 \ell_{e} & -\ell_{e}^{2} & -3 \ell_{e} & 4 \ell_{e}^{2}
\end{array}\right]
$$

The sensor output, $y$, is the sum of the contributions of the elements given by:

$$
y=\sum_{e} y_{e}=\mathbf{f}^{\top} \mathbf{C}_{s} \mathbf{q} .
$$

Here the element matrices have been assembled into the global matrix $\mathbf{C}_{s}$, in the usual way. The element nodal displacements, $w_{e i}$, have been incorporated into the global displacement vector $\mathbf{q}$, and the sensor nodal widths $f_{e i}$ have been assembled into a global vector $\mathbf{f}$. However, the sensor nodal widths at the clamped or pinned boundary conditions are not set to zero, whereas the corresponding displacements are set to zero. Thus in general $\mathbf{C}_{s}$ is a rectangular matrix. Comparing Equations (2) and (10), it is clear that:

$$
\mathbf{C}=\mathbf{f}^{\top} \mathbf{C}_{s} .
$$

\section{MODAL SENSORS FOR THE BASELINE SYSTEM}

In this section we review the derivation of the modal sensors for the baseline (deterministic) system. In general a linear dynamical system of the form (1) is expected to have complex modes (see, e.g., Adhikari, 1999, 2004b). Here proportional damping will be assumed so that the mode shapes of Equation (1) are real, and equal to the mode shapes of the undamped system. For light damping this approximation will introduce small errors. The mode shapes, $\boldsymbol{\Phi}$, are assumed to be normalized arbitrarily so that the modal mass is:

$$
\boldsymbol{\Phi}^{\top} \mathbf{M} \boldsymbol{\Phi}=\mathbf{M}_{m} .
$$

Applying the transformation to modal co-ordinates, $\mathbf{q}=\boldsymbol{\Phi} \mathbf{p}$, to Equation (1), gives:

$$
\begin{gathered}
\ddot{\mathbf{p}}+2 \mathbf{Z} \Omega \dot{\mathbf{p}}+\Omega^{2} \mathbf{p}=\mathbf{M}_{m}^{-1} \boldsymbol{\Phi}^{\top} \mathbf{B} \mathbf{u}, \\
\mathbf{y}=\mathbf{C} \boldsymbol{\Phi} \mathbf{p}=\mathbf{C}_{\mathbf{p}} \mathbf{p}
\end{gathered}
$$

Where

$$
\Omega=\operatorname{diag}\left[\omega_{1}, \omega_{2}, \ldots, \omega_{n}\right],
$$

is a diagonal matrix of the natural frequencies:

$$
\boldsymbol{\Phi}=\operatorname{diag}\left[\phi_{1}, \phi_{2}, \ldots, \phi_{n}\right],
$$

is a $n \times n$ matrix of mode shapes and:

$$
\mathbf{Z}=\operatorname{diag}\left[\zeta_{1}, \zeta_{2}, \ldots, \zeta_{n}\right],
$$

is a diagonal matrix of modal damping ratios. Thus, using Equation (11):

$$
\mathbf{C}_{\mathbf{p}}=\mathbf{C} \boldsymbol{\Phi}=\mathbf{f}^{\top} \mathbf{C}_{S} \boldsymbol{\Phi} .
$$

The modal sensor design problem is then to determine the sensor shape, defined by $\mathbf{f}$, to give the required modal output gain matrix, $\mathbf{C}_{\mathbf{p}}$. Clearly the modes must be scaled in some consistent manner, since otherwise for a particular set of output gains the modal output gain matrix will change depending on the mode scaling. This is particularly important when more than one mode is measured. Usually the number of elements describing the sensor shape is large and so Equation (18) will be underdetermined. In this case the pseudo inverse solution will produce the minimum norm solution.

An alternative is to minimize transducer curvature, while ensuring zero sensitivity to unwanted modes (Friswell, 2001). The minimum curvature ensures that the transducer may be manufactured as easily as possible. Thus, we wish to minimize:

$$
J_{c}(\mathbf{f})=\sum_{e} \int_{0}^{\ell_{e}} f_{e}^{\prime \prime}(\xi)^{2} \mathrm{~d} \xi=\sum_{e}\left\{\begin{array}{l}
f_{e 1} \\
f_{e 2} \\
f_{e 3} \\
f_{e 4}
\end{array}\right\}^{\top} \mathbf{H}_{e}\left\{\begin{array}{l}
f_{e 1} \\
f_{e 2} \\
f_{e 3} \\
f_{e 4}
\end{array}\right\},
$$


where

$$
\mathbf{H}_{e i j}=\int_{0}^{\ell_{e}} N_{e i}^{\prime \prime}(\xi) N_{e j}^{\prime \prime}(\xi) \mathrm{d} \xi
$$

$\mathbf{H}_{e}$ is equivalent to the element stiffness matrix with a unit flexural rigidity. Assembling the contributions from all of the elements gives:

$$
J_{c}(\mathbf{f})=\mathbf{f}^{\top} \mathbf{H} \mathbf{f}
$$

where $\mathbf{H}$ contains the element matrices, $\mathbf{H}_{e}$, and is symmetric. The sensor design problem then requires that $J_{c}$ is minimized, subject to the constraints given by Equation (18). This problem may be solved using Lagrange multipliers (Friswell, 2001), as the solution of:

$$
\left[\begin{array}{cc}
2 \mathbf{H} & \mathbf{C}_{s} \boldsymbol{\Phi} \\
\boldsymbol{\Phi}^{\top} \mathbf{C}_{s}^{\top} & \mathbf{0}
\end{array}\right]\left\{\begin{array}{l}
\mathbf{f} \\
\lambda
\end{array}\right\}=\left\{\begin{array}{c}
\mathbf{0} \\
\mathbf{C}_{\mathbf{p}}^{\top}
\end{array}\right\}
$$

where $\lambda$ is the vector of Lagrange multipliers.

\section{MODAL SENSORS FOR UNCERTAIN SYSTEMS}

The essential requirement for modal sensors is to enforce the constraints given by Equation (18) for the modes of interest. In most practical cases the number of constraints is fewer than the number of parameters determining the sensor shape and hence other metrics may be optimized, for example the sensor curvature outlined above. The development thus far has only considered a deterministic system. In reality the physical system will be uncertain, meaning that the mode shapes will be stochastic. One possibility is to use the freedom available in designing the sensor shape to ensure robustness to the system uncertainty for the modal sensors.

Consider the terms in Equation (18), repeated here for convenience:

$$
\mathbf{C}_{\mathbf{p}}=\mathbf{f}^{\top} \mathbf{C}_{S} \boldsymbol{\Phi}(\theta) .
$$

Note that the effect of system uncertainty is reflected by the random nature of the modal matrix $\boldsymbol{\Phi}(\theta)$.

$\mathbf{C}_{s}$ is determined from the element shape functions and is therefore fixed for a given mesh and will not change with the system parameters. Clearly the mode shapes of interest, $\boldsymbol{\Phi}$, will vary with the uncertain parameters. The vector $\mathbf{f}$ determines the shape of the sensor, and is obtained from the system optimization. Here we will assume this vector is deterministic, although of course implementing a required sensor shape in practice will be subject to manufacturing errors not considered in this article. Thus, for a given sensor the system uncertainty will produce a stochastic modal output vector $\mathbf{C}_{\mathbf{p}}$. Suppose the desired modal output vector is denoted $\mathbf{C}_{\mathbf{p} d}$.
Then to ensure the correct modal response we will enforce the constraint:

$$
\mathrm{E}\left[\mathbf{C}_{\mathbf{p}}\right]=\mathbf{C}_{\mathbf{p} d}=\mathbf{f}^{\top} \mathbf{C}_{s} \mathrm{E}[\boldsymbol{\Phi}(\theta)],
$$

where $\mathrm{E}[]$ denotes the expected value. Assuming that the mode shapes have mean $\boldsymbol{\Phi}_{0}$, we have:

$$
\mathbf{C}_{\mathbf{p} d}=\mathbf{f}^{\top} \mathbf{C}_{s} \boldsymbol{\Phi}_{0} .
$$

To ensure robustness we will minimize the sum of the variances of the modal outputs given by:

$$
J_{s}=\mathrm{E}\left[\left(\mathbf{C}_{\mathbf{p}}-\mathbf{C}_{\mathbf{p} d}\right)\left(\mathbf{C}_{\mathbf{p}}-\mathbf{C}_{\mathbf{p} d}\right)^{\top}\right] .
$$

The above choice, possibly the simplest, is clearly not unique. The motivation behind this choice arises from the need to minimize the non-zero second-order effect due to uncertainty. One can also consider a weighted approach and minimize $\mathrm{E}\left[\left(\mathbf{C}_{\mathbf{p}}-\mathbf{C}_{\mathbf{p} d}\right) \mathbf{W}\left(\mathbf{C}_{\mathbf{p}}-\mathbf{C}_{\mathbf{p} d}\right)^{\top}\right]$, where $\mathbf{W}$ is a suitable weighting matrix. The expression in Equation (26) is equivalent to using equal weight for all the terms. Another alternative could be to generate the full covariance matrix between different modal outputs and perhaps minimize some (matrix) norm of the full covariance matrix $\left(\mathbf{C}_{\mathbf{p}}-\mathbf{C}_{\mathbf{p} d}\right) \otimes\left(\mathbf{C}_{\mathbf{p}}-\mathbf{C}_{\mathbf{p} d}\right)$ (where $\otimes$ denotes the Kroneker product). This will be more demanding case and would involve the solution of a very large $n^{2}$ dimensional matrix optimization problem.

Recalling that the mode shapes have mean $\boldsymbol{\Phi}_{0}$, the required optimization is to minimize:

$$
J_{s}=\mathbf{f}^{\top} \mathbf{C}_{s} \mathrm{E}\left[\left(\boldsymbol{\Phi}-\boldsymbol{\Phi}_{0}\right)\left(\boldsymbol{\Phi}-\boldsymbol{\Phi}_{0}\right)^{\top}\right] \mathbf{C}_{s}^{\top} \mathbf{f},
$$

with the constraint:

$$
\mathbf{C}_{\mathbf{p} d}=\mathbf{f}^{\top} \mathbf{C}_{S} \mathbf{\Phi}_{0} .
$$

This optimization is equivalent to minimizing the sensor curvature, discussed in the previous section, with:

$$
\mathbf{H}=\mathbf{C}_{s} \mathrm{E}\left[\left(\boldsymbol{\Phi}-\boldsymbol{\Phi}_{0}\right)\left(\boldsymbol{\Phi}-\boldsymbol{\Phi}_{0}\right)^{\top}\right] \mathbf{C}_{s}^{\top} .
$$

The calculation of the above quantity requires the calculation of second-order statistical properties of the mode shapes and is discussed in the next section.

\section{STOCHASTIC FINITE ELEMENTS FOR BEAMS WITH PARAMETRIC UNCERTAINTY}

\section{Spectral Decomposition of Random Fields}

Suppose $F(\mathbf{r}, \theta)$ is a random field with a covariance function $C_{H}\left(\mathbf{r}_{1}, \mathbf{r}_{2}\right)$ defined in a space $\mathcal{D}$. Here $\theta$ denotes an element of the (random) sample space $\Omega$ so that $\theta \in \Omega$. Mathematically and numerically it is very difficult 
to deal with random fields directly in the equations of motion which are often expressed by partial differential equations. For this reason it is required to discretize a random field in terms of random variables. Once this is done, then a wide range of mathematical and numerical techniques can be used to solve the resulting discrete stochastic differential equations. Among the many discretization techniques, the spectral decomposition of random fields using the Karhunen-Loève expansion turns out to be very useful in practice. In this article this approach has been taken to obtain the optimal shape of the distributed sensors.

Since the covariance function is finite, symmetric and positive definite it can be represented by a spectral decomposition. Using this spectral decomposition, the random field $F(\mathbf{r}, \theta)$ can be expressed in a generalized Fourier type of series as:

$$
F(\mathbf{r}, \theta)=F_{0}(\mathbf{r})+\sum_{j=1}^{\infty} \sqrt{\lambda_{j}} \xi_{j}(\theta) \varphi_{j}(\mathbf{r}),
$$

where $\xi_{j}(\theta)$ are uncorrelated random variables and throughout the article $(\bullet)_{0}$ implies the deterministic part corresponding to $(\bullet)$. The constants $\lambda_{j}$ and functions $\varphi_{j}(\mathbf{r})$ are eigenvalues and eigenfunctions satisfying the integral equation:

$$
\int_{\mathcal{D}} C_{F}\left(\mathbf{r}_{1}, \mathbf{r}_{2}\right) \varphi_{j}\left(\mathbf{r}_{1}\right) \mathrm{d} \mathbf{r}_{1}=\lambda_{j} \varphi_{j}\left(\mathbf{r}_{2}\right), \quad \forall i=1,2, \ldots
$$

The spectral decomposition in Equation (30), which discretizes a random field into random variables, is known as the Karhunen-Loève expansion. The series in Equation (30) can be ordered in a decreasing series so that it can be truncated after a finite number of terms with a desired accuracy. We refer the books by Ghanem and Spanos (1991), Papoulis and Pillai (2002), and references therein for further discussions on Karhunen-Loève expansion.

In this article 1D systems are considered. To demonstrate the approach a Gaussian random field with an exponentially decaying autocorrelation function is considered. Such a model is representative of many physical systems and closed-form expressions for the Karhunen-Loève expansion may be obtained. The autocorrelation function can be expressed as:

$$
C\left(x_{1}, x_{2}\right)=e^{-\left|x_{1}-x_{2}\right| / b} .
$$

Here the constant $b$ is known as the correlation length and it plays an important role in the description of a random field. If the correlation length is very small, then the random field becomes close to a delta-correlated field, often known as white noise. If the correlation length is very large compared to domain under consideration, then the random field effectively becomes a random variable. Assuming the mean is zero, then the underlying random field $F(x, \theta)$ can be expanded using the Karhunen-Loève expansion (Ghanem and Spanos, 1991; Papoulis and Pillai, 2002) in the interval $-a \leq x \leq a$ as:

$$
F(x, \theta)=\sum_{j=1}^{\infty} \xi_{j}(\theta) \sqrt{\lambda_{j}} \varphi_{j}(x) .
$$

Using the notation $c=1 / b$, the corresponding eigenvalues and eigenfunctions, for odd $j$ are given by:

$$
\begin{aligned}
& \lambda_{j}=\frac{2 c}{\omega_{j}^{2}+c^{2}}, \quad \varphi_{j}(x)=\frac{\cos \left(\omega_{j} x\right)}{\sqrt{a+\sin \left(2 \omega_{j} a\right) / 2 \omega_{j}}}, \\
& \text { where } \tan \left(\omega_{j} a\right)=\frac{c}{\omega_{j}},
\end{aligned}
$$

and for even $j$ are given by:

$$
\begin{aligned}
& \lambda_{j}=\frac{2 c}{\omega_{j}^{2}+c^{2}}, \quad \varphi_{j}(x)=\frac{\sin \left(\omega_{j} x\right)}{\sqrt{a-\sin \left(2 \omega_{j} a\right) / 2 \omega_{j}}}, \\
& \text { where } \tan \left(\omega_{j} a\right)=\frac{\omega_{j}}{-c} .
\end{aligned}
$$

These eigenvalues and eigenfunctions will now be used to obtain the element mass and stiffness matrices.

It should be noted that it is perfectly possible to have correlation functions other than the exponential correlation function considered in Equation (32). The actual functional form of the correlation function is determined from the real data concerning the underlying random process. For other functional forms of the correlation function, the integral Equation (31) will be different. Consequently, the expressions of the eigenvalues and eigenfunctions in Equations (34) and (35) will also be different. As an example, we refer to the book by Ghanem and Spanos (1991) (Chapter 2) for the eigenvalues and eigenfunctions with a triangular correlation function. The choice of the correlation function will affect the shape of the sensors, but it is beyond the scope of this work to investigate this issue in detail. The formulation to be followed in the rest of the article assumes the validity of the exponential correlation function for the uncertain system properties.

\section{Element Matrices for the Stochastic Beam}

The equation of motion of an undamped Euler-Bernoulli beam of length $L$ with random bending stiffness and mass distribution can be expressed as:

$$
\frac{\partial^{2}}{\partial x^{2}}\left[E I(x, \theta) \frac{\partial^{2} Y(x, t)}{\partial x^{2}}\right]+\rho A(x, \theta) \frac{\partial^{2} Y(x, t)}{\partial t^{2}}=p(x, t) .
$$


Here $Y(x, t)$ is the transverse flexural displacement, $E I(x)$ is the flexural rigidity, $\rho A(x)$ is the mass per unit length, and $p(x, t)$ is the applied forcing. It is assumed that the bending stiffness, $E I$, and mass per unit length, $\rho A$, are random fields of the form:

$$
E I(x, \theta)=E I_{0}\left(1+\epsilon_{1} F_{1}(x, \theta)\right),
$$

and

$$
\rho A(x, \theta)=\rho A_{0}\left(1+\epsilon_{2} F_{2}(x, \theta)\right) .
$$

The subscript 0 indicates the mean values, $0<\epsilon_{i} \ll 1$ $(i=1,2)$ are deterministic constants and the random fields $F_{i}(x, \theta)$ are taken to have zero mean, unit standard deviation and covariance $R_{i j}(\xi)$. Since, $E I(x, \theta)$ and $\rho A(x, \theta)$ are strictly positive, $F_{i}(x, \theta) \quad(i=1,2)$ are required to satisfy the conditions $P[1+$ $\left.\epsilon F_{i}(x, \theta) \leq 0\right]=0$. This requirement, strictly speaking, rules out the use of Gaussian models for these random fields. However, for small $\epsilon_{i}$, it is expected that Gaussian models can still be used if the primary interest of the analysis is to estimate the first few response moments and not the response behavior near tails of the probability distributions.

For notational convenience, we express the shape functions in Equations (3) as:

$$
\mathbf{N}(x)=\Gamma \mathbf{s}(x),
$$

where

$$
\boldsymbol{\Gamma}=\left[\begin{array}{cccc}
1 & 0 & \frac{-3}{\ell_{e}^{2}} & \frac{2}{\ell_{e}^{3}} \\
0 & 1 & \frac{-2}{\ell_{e}^{2}} & \frac{1}{\ell_{e}^{2}} \\
0 & 0 & \frac{3}{\ell_{e}^{2}} & \frac{-2}{\ell_{e}^{3}} \\
0 & 0 & \frac{-1}{\ell_{e}^{2}} & \frac{1}{\ell_{e}^{2}}
\end{array}\right] \text { and } \mathbf{s}(x)=\left[1, x, x^{2}, x^{3}\right]^{T} .
$$

Using the expression for $E I(x, \theta)$ given by Equation (37), the element stiffness matrix can be obtained as:

$$
\begin{aligned}
\mathbf{K}_{e}(\theta) & =\int_{0}^{\ell_{e}} \mathbf{N}^{\prime \prime}(x) E I(x, \theta) \mathbf{N}^{\prime \prime T}(x) \mathrm{d} x \\
& =\int_{0}^{\ell_{e}} E I_{0}\left(1+\epsilon_{1} F_{1}(x, \theta)\right) \mathbf{N}^{\prime \prime}(x) \mathbf{N}^{\prime \prime T}(x) \mathrm{d} x .
\end{aligned}
$$

Expanding the random field $F_{1}(x, \theta)$ in the Karhunen-Loève spectral decomposition (33) we have:

$$
\mathbf{K}_{e}(\theta)=\mathbf{K}_{e_{0}}+\Delta \mathbf{K}_{e}(\theta),
$$

where the deterministic part is given by:

$$
\mathbf{K}_{e_{0}}=E I_{0} \int_{0}^{\ell_{e}} \mathbf{N}^{\prime \prime}(x) \mathbf{N}^{\prime \prime T}(x) \mathrm{d} x .
$$

and the random part is given by:

$$
\Delta \mathbf{K}_{e}(\theta)=\epsilon_{1} \sum_{j=1}^{N_{\mathrm{K}}} \xi_{\mathrm{K} j}(\theta) \sqrt{\lambda_{\mathrm{K} j}} \mathbf{K}_{e j} .
$$

The constant $N_{\mathrm{K}}$ is the number of terms retained in the Karhunen-Loève expansion and $\xi_{\mathrm{K} j}(\theta)$ are uncorrelated Gaussian random variables with zero mean and unit standard deviation. The constant matrices $\mathbf{K}_{e_{i}}$ can be expressed as:

$$
\mathbf{K}_{e j}=E I_{0} \int_{0}^{\ell_{e}} \varphi_{\mathrm{K} j}\left(x_{e}+x\right) \mathbf{N}^{\prime \prime}(x) \mathbf{N}^{\prime \prime T}(x) \mathrm{d} x,
$$

where $x_{e}$ denotes the starting coordinate of the element $e$. The functions $\varphi_{\mathrm{K} j}$ are as defined in Equations (34) and (35). Closed-form expressions of these matrices are derived in section A.1 in the Appendix.

Using the same approach, the mass matrix can be obtained as:

$$
\mathbf{M}_{e}(\theta)=\mathbf{M}_{e_{0}}+\Delta \mathbf{M}_{e}(\theta),
$$

where the deterministic part is given by:

$$
\mathbf{M}_{e_{0}}=\rho A_{0} \int_{0}^{\ell_{e}} \mathbf{N}(x) \mathbf{N}^{T}(x) \mathrm{d} x .
$$

and the random part is given by:

$$
\Delta \mathbf{M}_{e}(\theta)=\epsilon_{2} \sum_{j=1}^{N_{\mathrm{M}}} \xi_{\mathrm{M} j}(\theta) \sqrt{\lambda_{\mathrm{M} j}} \mathbf{M}_{e j} .
$$

The constant $N_{\mathrm{M}}$ is the number of terms retained in Karhunen-Loève expansion and the constant matrices $\mathbf{M}_{e_{j}}$ can be expressed as:

$$
\mathbf{M}_{e j}=\rho A_{0} \int_{0}^{\ell_{e}} \varphi_{\mathrm{M} j}\left(x_{e}+x\right) \mathbf{N}(x) \mathbf{N}^{T}(x) \mathrm{d} x .
$$

The closed-form expressions of these matrices are given in section A.2 in the Appendix. Using the conventional approach, these element matrices can be assembled to form the global random stiffness and mass matrices of the form:

$$
\mathbf{K}(\theta)=\mathbf{K}_{0}+\Delta \mathbf{K}(\theta)
$$

and

$$
\mathbf{M}(\theta)=\mathbf{M}_{0}+\Delta \mathbf{M}(\theta)
$$


Here the deterministic parts $\mathbf{K}_{0}$ and $\mathbf{M}_{0}$ are the usual global stiffness and mass matrices obtained form the conventional finite element method. The random parts can be expressed as:

$$
\begin{aligned}
& \Delta \mathbf{K}(\theta)=\epsilon_{1} \sum_{j=1}^{N_{\mathrm{K}}} \xi_{\mathrm{K}_{j}}(\theta) \sqrt{\lambda_{\mathrm{K}_{j}}} \mathbf{K}_{j}, \\
& \text { and } \quad \Delta \mathbf{M}(\theta)=\epsilon_{2} \sum_{j=1}^{N_{\mathrm{M}}} \xi_{\mathrm{M}_{j}}(\theta) \sqrt{\lambda_{\mathrm{M}_{j}}} \mathbf{M}_{j},
\end{aligned}
$$

and the element matrices $\mathbf{K}_{e j}$ and $\mathbf{M}_{e j}$ have been assembled into the global matrices $\mathbf{K}_{j}$ and $\mathbf{M}_{j}$. The total number of random variables depend on the number of terms used for the truncation of the infinite series, Equation (33). This in turn depends on the respective correlation lengths of the underlying random fields; the smaller the correlation length, the higher the number of terms required and vice versa.

\section{EIGENSOLUTION STATISTICS OF STOCHASTIC SYSTEMS}

The estimation of the means of the mode shapes and the $\mathbf{H}$ matrix in Equation (29) requires the solution of a random eigenvalue problem for linear stochastic dynamical systems. Specifically, in this section we propose explicit expressions to obtain $\mathrm{E}[\boldsymbol{\Phi}(\theta)]$ and elements of the matrix:

$$
\mathbf{W}=\mathrm{E}\left[\left(\boldsymbol{\Phi}(\theta)-\boldsymbol{\Phi}_{0}\right)\left(\boldsymbol{\Phi}(\theta)-\boldsymbol{\Phi}_{0}\right)^{\top}\right],
$$

for linear dynamical systems with uncertain properties modeled by random fields.

One needs to solve random eigenvalue problems to obtain statistical properties of the eigenvalues and eigenvectors appearing in stochastic dynamical systems. Several studies have been conducted on this topic since the mid-sixties. The paper by Boyce (1968), the book by Scheidt and Purkert (1983) and the review papers by Ibrahim (1987), Benaroya (1992), Manohar and Ibrahim (1999), and Manohar and Gupta (2003) are useful sources of information on early work in this area of research and also provide a systematic account of different approaches to random eigenvalue problems. The central aim of studying random eigenvalue problems is to obtain the joint probability density function of the eigenvalues and the eigenvectors. The current literature on random eigenvalue problems arising in engineering systems is dominated by the mean-centered perturbation methods, see for example Collins and Thomson (1969), Hasselman and Hart (1972), Hart (1973), Ramu and Ganesan (1992a,b, 1993a,b), Sankar et al. (1993), Song et al. (1995), den Nieuwenhof and
Coyette (2003). These methods work well when the uncertainties are small and the parameter distribution is Gaussian.

Some authors have proposed methods which are not based on mean-centered perturbation method. Grigoriu (1992) examined the roots of characteristic polynomials of real symmetric random matrices using the distribution of zeros of random polynomials. Lee and Singh (1994) proposed a direct matrix product (Kronecker product) method to obtain the first two moments of the eigenvalues of discrete linear systems. Nair and Keane (2003) proposed a stochastic reduced basis approximation which can be applied to discrete or discretized continuous dynamic systems. Hála (1994) and Mehlhose et al. (1999) used a Ritz method to obtain closed-form expressions for moments and probability density functions of the eigenvalues (in terms of Chebyshev-Hermite polynomials). Ghosh et al. (2005) used a polynomial chaos expansion for random eigenvalue problems. Adhikari (2004a) considered complex random eigenvalue problems associated with nonproportionally damped systems. Verhoosel et al. (2006) proposed an iterative method that can be applied to non-symmetric random matrices also. Rahman (2006) developed a dimensional decomposition method which does not require the calculation of eigensolution derivatives. Adhikari $(2006,2007)$ and Adhikari and Friswell (2007) developed an asymptotic approach and an optimal series-expansion method to obtain second and higher order joint statistics of eigenvalues. Here, the first-order perturbation approach is proposed to calculate the second-order statistics appearing in Equation (29).

The random eigenvalue problem corresponding to an undamped stochastic system can be expressed as:

$$
\left[\mathbf{K}_{0}+\Delta \mathbf{K}(\theta)\right] \phi_{j}=\omega_{j}^{2}\left[\mathbf{M}_{0}+\Delta \mathbf{M}(\theta)\right] \phi_{j} .
$$

Using the first-order perturbation method, each eigenvector can be expressed as:

$$
\phi_{j} \approx \phi_{0_{j}}+\sum_{k=1}^{N_{\mathrm{K}}} \frac{\partial \phi_{j}}{\partial \xi_{\mathrm{K}_{k}}} \xi_{\mathrm{K}_{k}}(\theta)+\sum_{k=1}^{N_{\mathrm{M}}} \frac{\partial \phi_{j}}{\partial \xi_{\mathrm{M}_{k}}} \xi_{\mathrm{M}_{k}}(\theta) .
$$

The derivative of the eigenvectors can be obtained using the expression derived by Fox and Kapoor (1968) as:

$$
\begin{aligned}
\frac{\partial \phi_{j}}{\partial \xi_{\mathrm{K}_{k}}} & =\sum_{r=1, r \neq j}^{n} a_{j k r} \phi_{0_{r}} \\
\text { where } a_{j k r} & =\epsilon_{1} \sqrt{\lambda_{\mathrm{K}_{k}}} \frac{\phi_{0_{r}}^{T} \mathbf{K}_{k} \phi_{0_{j}}}{\omega_{0_{j}}^{2}-\omega_{0_{r}}^{2}},
\end{aligned}
$$


and

$$
\begin{aligned}
\frac{\partial \phi_{j}}{\partial \xi_{\mathrm{M}_{k}}} & =\sum_{r=1}^{n} a_{j k r} \phi_{0_{r}} \\
\text { where } \quad a_{j k r} & =-\epsilon_{2} \sqrt{\lambda_{\mathrm{M}_{k}}} \omega_{0_{j}}^{2} \frac{\phi_{0_{r}}^{T} \mathbf{M}_{k} \phi_{0_{j}}}{\omega_{0_{j}}^{2}-\omega_{0_{r}}^{2}} \forall r \neq j ; \\
a_{j k j} & =-\epsilon_{2} \frac{1}{2} \sqrt{\lambda_{\mathrm{M}_{k}}} \omega_{0_{j}}^{2}\left(\phi_{0_{j}}^{T} \mathbf{M}_{k} \phi_{0_{j}}\right) .
\end{aligned}
$$

In the above expressions $\omega_{0_{j}}$ and $\phi_{0_{j}}$ correspond to the natural frequency and mode shape of the underlying baseline system. From the expansion in Equation (56) it can be seen that the mean of the mode shapes are simply the mode shapes of the corresponding baseline system. Combining the preceding three equations, we can express:

$$
\phi_{j} \approx \phi_{0_{j}}+\mathbf{A}^{(j)} \xi(\theta),
$$

where the $n \times\left(N_{\mathrm{K}}+N_{\mathrm{M}}\right)$ coefficient matrix $\mathbf{A}^{(j)}$ and the $\left(N_{\mathrm{K}}+N_{\mathrm{M}}\right)$ dimensional random vector $\xi_{t}$ are defined as:

$$
\begin{aligned}
\mathbf{A}^{(j)} & =\left[\frac{\partial \phi_{j}}{\partial \xi_{\mathrm{K}_{k}}}, k=1,2, \ldots, N_{\mathrm{K}} \mid \frac{\partial \phi_{j}}{\partial \xi_{\mathrm{M}_{k}}}, k=1,2, \ldots, N_{\mathrm{M}}\right] \\
\text { and } \boldsymbol{\xi} & =\left[\begin{array}{c}
\xi_{\mathrm{K}_{k}} \\
\xi_{\mathrm{M}_{k}}
\end{array}\right] .
\end{aligned}
$$

To obtain the elements of the $\mathbf{W}$ matrix in Equation (54), we define:

$$
\delta \boldsymbol{\Phi}=\boldsymbol{\Phi}(\theta)-\boldsymbol{\Phi}_{0},
$$

for notational convenience. From Equations (54) and (59) we have:

$$
\begin{aligned}
W_{k l} & =\sum_{j=1}^{n} \mathrm{E}\left[\delta \Phi_{k j} \delta \Phi_{l j}\right] \\
& =\sum_{j=1}^{n} \mathrm{E}\left[\sum_{r=1}^{N_{\mathrm{K}}+N_{\mathrm{M}}} A_{k r}^{(j)} \xi_{r}(\theta) \sum_{s=1}^{N_{\mathrm{K}}+N_{\mathrm{M}}} A_{l s}^{(j)} \xi_{s}(\theta)\right] \\
& =\sum_{j=1}^{n} \sum_{r=1}^{N_{\mathrm{K}}+N_{\mathrm{M}}} A_{k r}^{(j)} \sum_{s=1}^{N_{\mathrm{K}}+N_{\mathrm{M}}} A_{l s}^{(j)} \mathrm{E}\left[\xi_{r}(\theta) \xi_{s}(\theta)\right] .
\end{aligned}
$$

Recalling that all of the random variables are uncorrelated, we have $\mathrm{E}\left[\xi_{r}(\theta) \xi_{s}(\theta)\right]=\delta_{r s}$. Using this, the expression in Equation (62) can be simplified to:

$$
W_{k l}=\sum_{j=1}^{n} \sum_{s=1}^{N_{\mathrm{K}}+N_{\mathrm{M}}} A_{k s}^{(j)} A_{l s}^{(j)} .
$$

The elements of $A_{k r}^{(j)}$ are completely defined by Equations (57) and (58).

\section{NUMERICAL EXAMPLE}

A clamped-clamped beam example (Gawronski, 2000; Friswell, 2001) will be used to demonstrate the design of modal sensors and the effect of system uncertainty. The baseline model of the steel beam has length $300 \mathrm{~mm}$ and cross-section $20 \times 5 \mathrm{~mm}^{2}$. Bending in the more flexible plane is modeled by using 15 finite elements. Models with 30 and 60 elements were used to check that the matrix $\mathbf{W}$ in Equation (54) and the sensor shapes converge with the number of elements, subject to the numerical conditioning issues discussed later. In this study damping is assumed to be $1 \%$ in all modes. The first six natural frequencies for the baseline beam are 295.4, 814.2, 1596.5, 2639.9, 3946.1, and $5517.5 \mathrm{~Hz}$. The force input is applied at node 7 for the sensor design. A sensor is designed by considering only the first five modes of the beam. The sensor gain constant is assumed to be unity, $K_{s}=1$, since it is most important to compute the sensor shape, rather than the calibration constant.

It is assumed that the bending stiffness $E I(x)$ and mass per unit length $\rho A(x)$ are random fields of the form given in Equations (37) and (38). $E I_{0}$ and $\rho A_{0}$ are the corresponding properties of the baseline model and the strength parameters are assumed to be $\epsilon_{1}=0.05$, and $\epsilon_{2}=0.075$. The random fields $F_{1}(x)$ and $F_{2}(x)$ are assumed to be homogenous Gaussian random fields with an exponential correlation function given by Equation (32). To illustrate the approach the correlation length is assumed to be 0.2 times the length of the beam.

The following two cases are considered:

- Case 1: sensor designed to excite only the first mode, with a peak in the receptance of $0.01 \mathrm{~m} / \mathrm{N}$.

- Case 2: sensor designed to excite modes three to five only, with equal peaks in the receptance of $0.01 \mathrm{~m} / \mathrm{N}$.

For each case, design for the deterministic and uncertain systems were considered.

Figure 1 shows the shape of the sensor designed to excite only the first mode of the underlying deterministic system, with a peak in the receptance of $0.01 \mathrm{~m} / \mathrm{N}$. The shape was obtained by minimizing the curvature, and the nodal width values used, together with the element shape functions, to produce a continuous sensor width function. The shape is very similar to the curvature of the first mode of the deterministic beam. Figure 2 shows the shape of the sensor designed to measure modes three to five of the underlying deterministic system. Notice that the shape is now much more complex.

Now we turn our attention to the stochastic system where the shapes of the sensors are obtained using two 

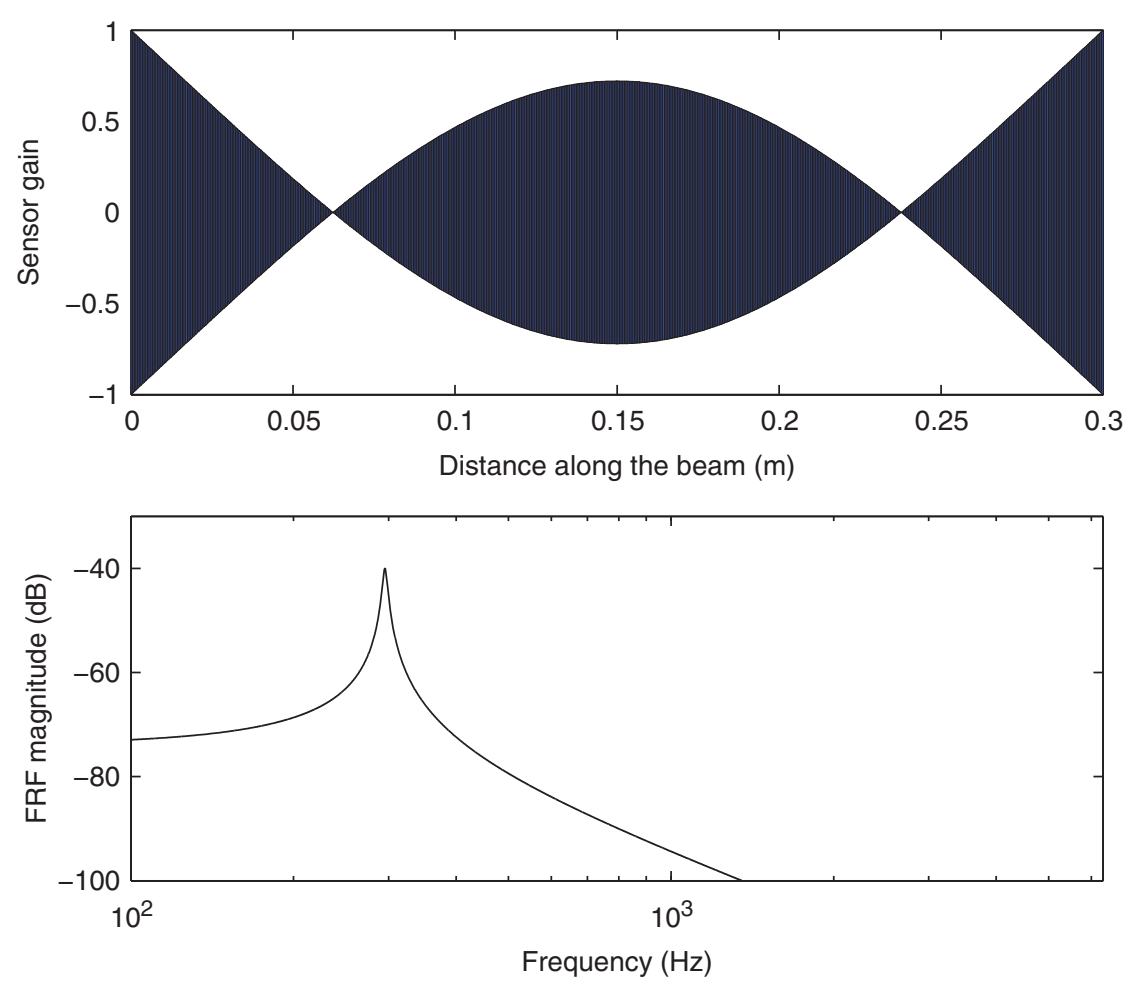

Figure 1. The distributed sensor shape to measure the first mode only for the baseline system and the associated receptance.
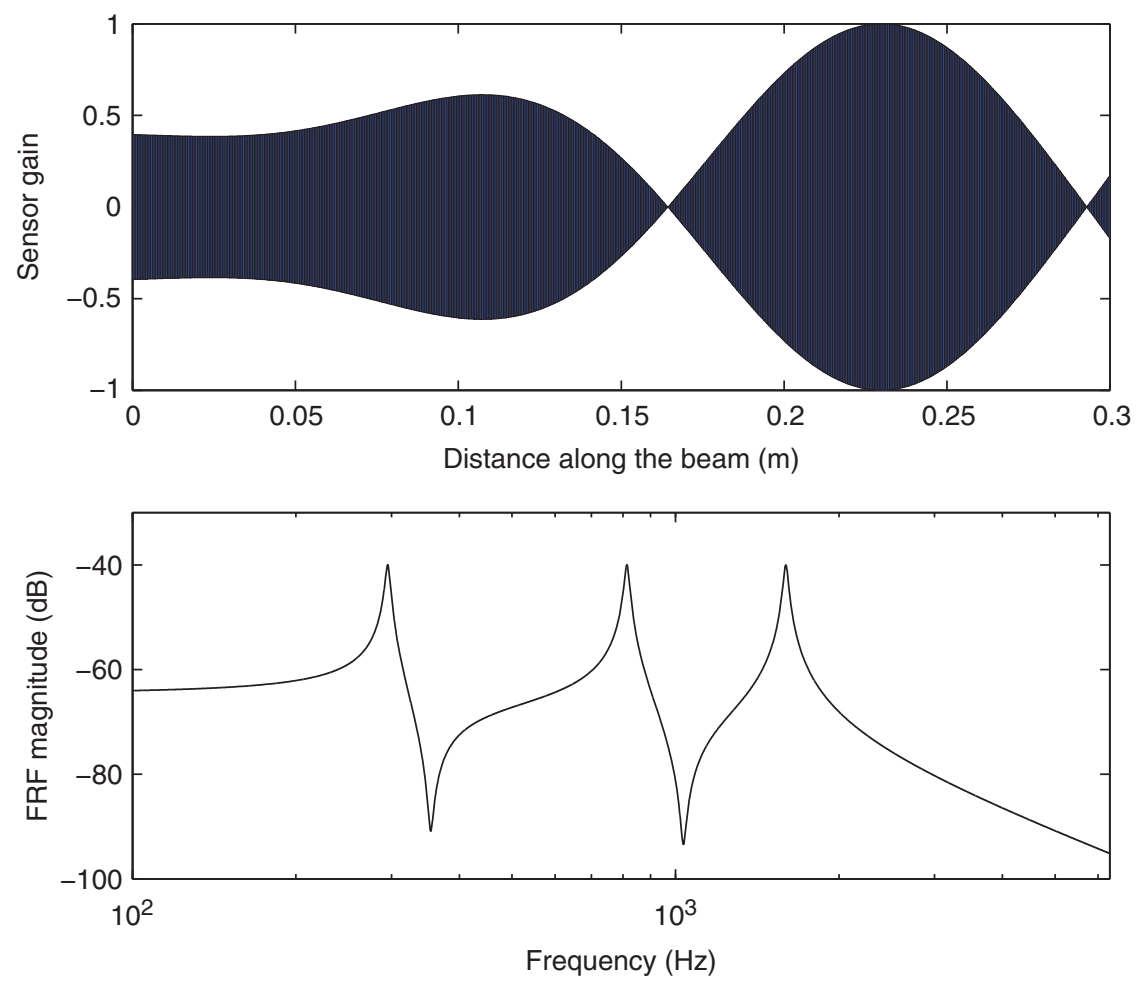

Figure 2. The distributed sensor shape to measure modes 3-5 only for the baseline system and the associated receptance. 


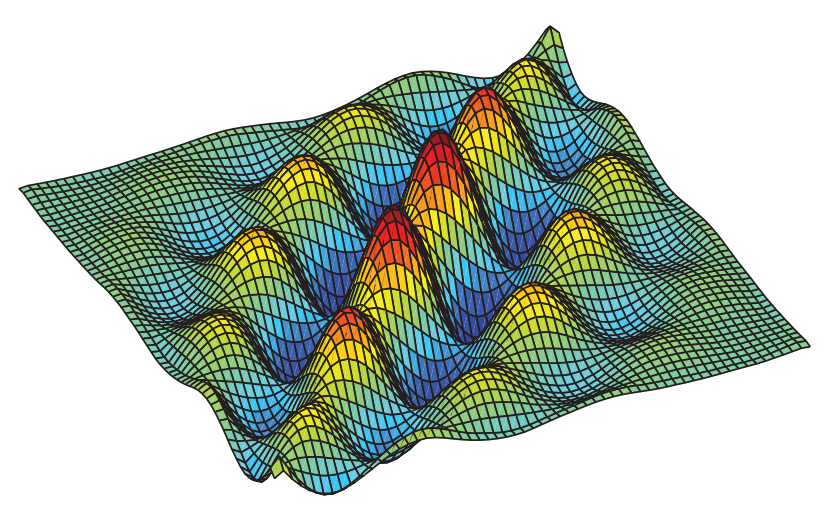

Figure 3. The translational elements of the matrix $H_{\mathrm{r}}$ for a beam model with 60 finite elements.

objective functions. The first objective is to minimize the curvature, given by Equation (21) as:

$$
J_{c}(\mathbf{f})=\mathbf{f}^{\top} \mathbf{H}_{c} \mathbf{f},
$$

where the subscript $c$ on the matrix $\mathbf{H}$ highlights that its origin is the curvature of the sensor. The second objective is based on robustness, and the function has the same form, that is,

$$
J_{r}(\mathbf{f})=\mathbf{f}^{\top} \mathbf{H}_{r} \mathbf{f},
$$

where the matrix $\mathbf{H}_{r}$ is now given by the system uncertainty, denoted by $\mathbf{H}$ in Equation (29). The translational elements of this matrix are shown Figure 3 for a beam model with 60 finite elements and this figure shows that the matrix is a smooth function of spatial position. The equivalent plots for models with 15 or 30 elements are very similar, but with fewer points, showing that the matrix $\mathbf{H}_{r}$ converges as the number of elements in the model increases; the matrix from the model with 60 elements is shown to highlight the detail in the matrix.

These two objective functions will be combined into the single objective:

$$
J(\mathbf{f})=\mu J_{c}(\mathbf{f})+(1-\mu) J_{r}(\mathbf{f}), \quad \text { for } 0 \leq \mu \leq 1 .
$$

For $\mu=1$ only the curvature is minimized, whereas for $\mu=0$ only the effect of the uncertainty is minimized. As $\mu$ varies the plot of $J_{c}$ against $J_{r}$ indicates the trade off between the two objectives, known as the Pareto front or curve, with the optimum value of $\mu$ near the corner of this curve. The form of the Pareto curve is very difficult to derive in closed form, although the regularization community (where it is known as the L-curve) have a detailed understanding of its properties (Hansen, 1992, 1994; Ahmadian et al., 1998; Titurus and Friswell, 2008). If the Pareto curve has a corner then this point is an optimum trade-off between the two objectives, and this corner may be calculated based on finding the point of maximum curvature (Hansen and O'Leary, 1993).

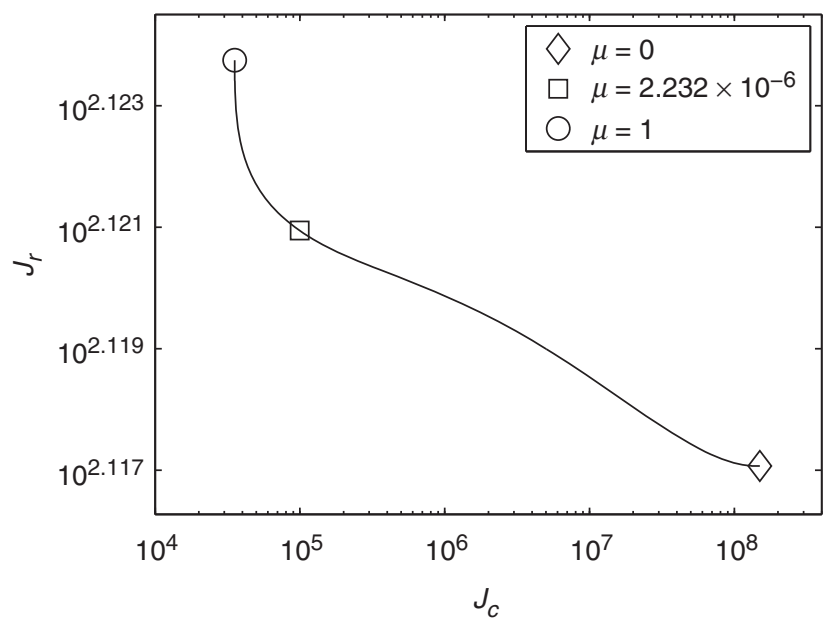

Figure 4. The Pareto curve for case 1, to measure the first mode only. The markers denote the points corresponding to the sensor shapes given in Figure 5.
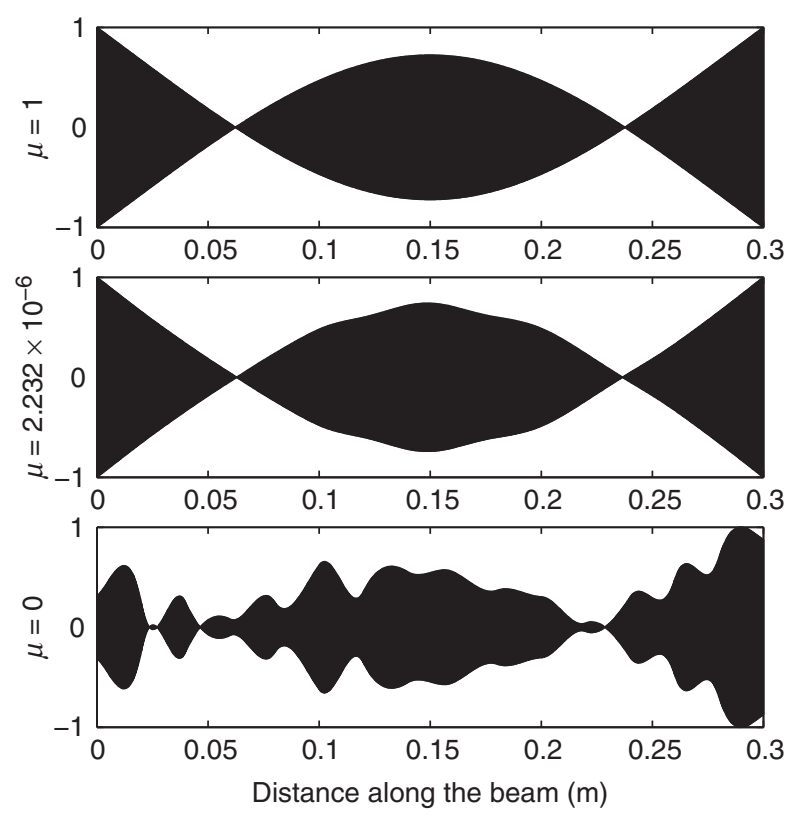

Figure 5. Three example distributed sensor shapes to measure the first mode only, case 1.

The Pareto curve for case 1 is shown in Figure 4 for a correlation length of $b=0.2 \mathrm{~L}$ for the finite element model with 15 elements. As expected the curve is monotonic. The sensor shapes for three values of $\mu$ marked on the Pareto curve are shown in Figure 5. The three values correspond to the extremes of the Pareto curve and a point close to the corner. For $\mu=1$ the system uncertainty has zero weight and hence the sensor shape is the same as the deterministic case shown in Figure 1. As expected this gives the lowest value of the smoothness objective function $J_{c}$. As $\mu$ is reduced the smoothness function value increases, but since more weight is given to the system uncertainty, the robustness objective function $J_{r}$ reduces. The optimal value of $\mu$ is near the 


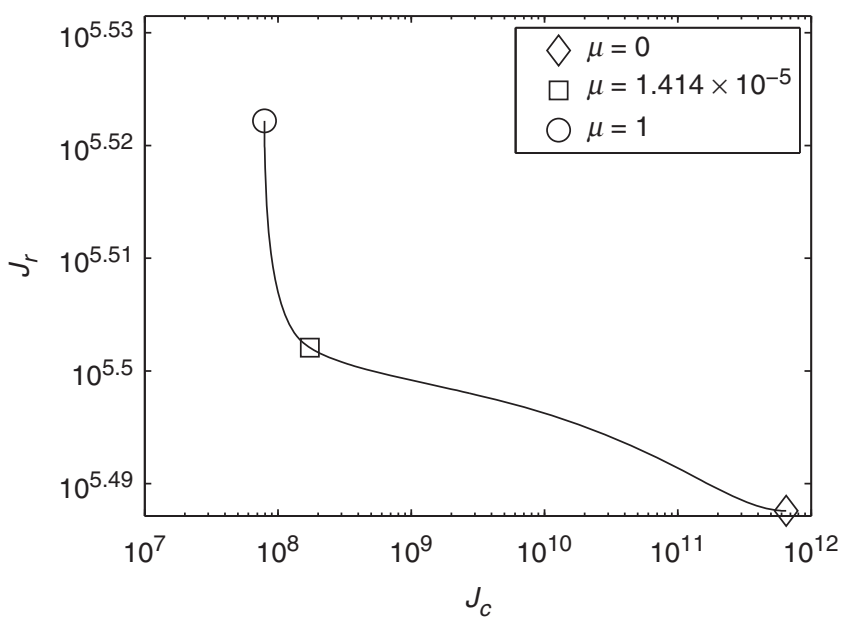

Figure 6. The Pareto curve for case 4, to measure modes three to five only. The markers denote the points corresponding to the sensor shapes given in Figure 7.
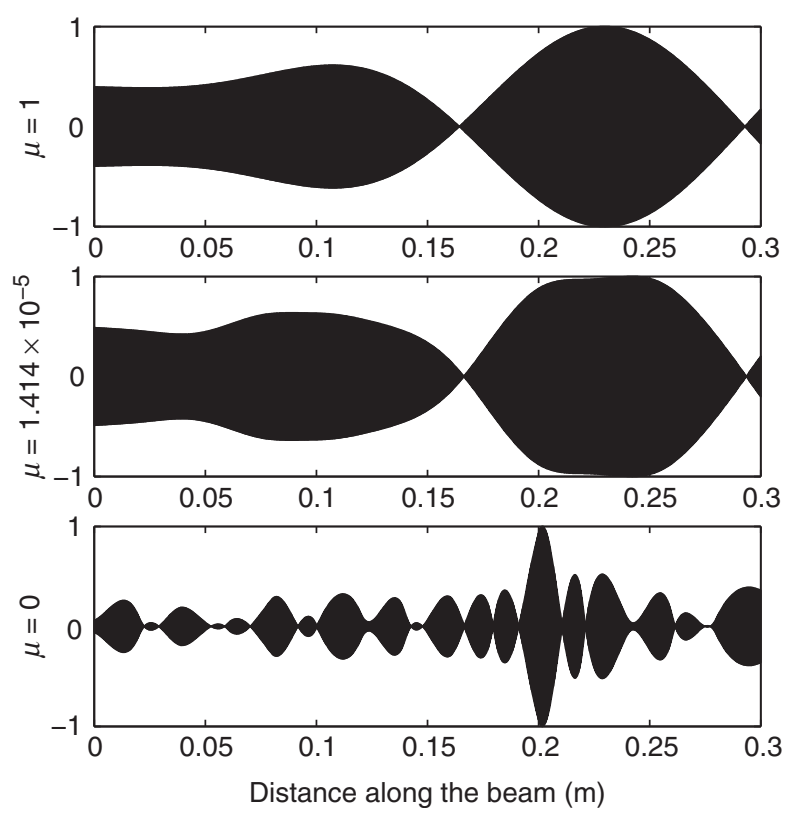

Figure 7. Three example distributed sensor shapes to measure modes 3-5 only, case 2.

corner of the Pareto curve, and the shape of the sensor at this point (shown in Figure 5) is close to the deterministic shape. It should be noted that the range of the objective functions are very different, because of the different definitions, but that any scaling will not affect the Pareto curve, merely the scaling of $\mu$. This is the reason why the value of $\mu$ at the corner is small. Notice that the robustness objective function is very insensitive to the value of $\mu$, which means that the smoothness objective is very good at rejecting the uncertainty in the system. When $\mu=0$ the design is based solely on the robustness objective, and the sensor shape is now much more complex and also the intuitive nature of the sensor shapes are less obvious.

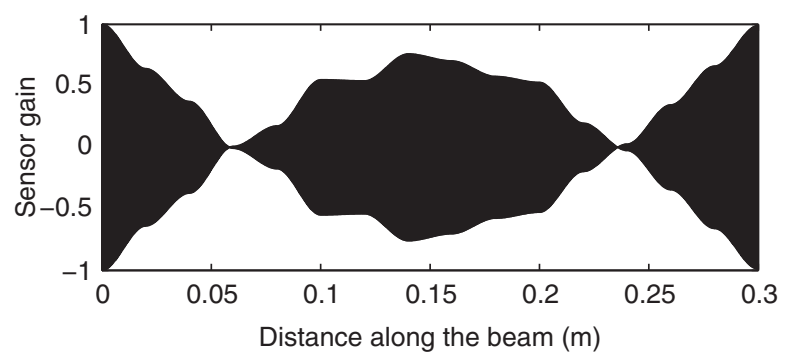

Figure 8. The sensor shape solely based on the robustness criterion for case 1 , but using a threshold of $10^{-6}$ on the singular values in the solution.

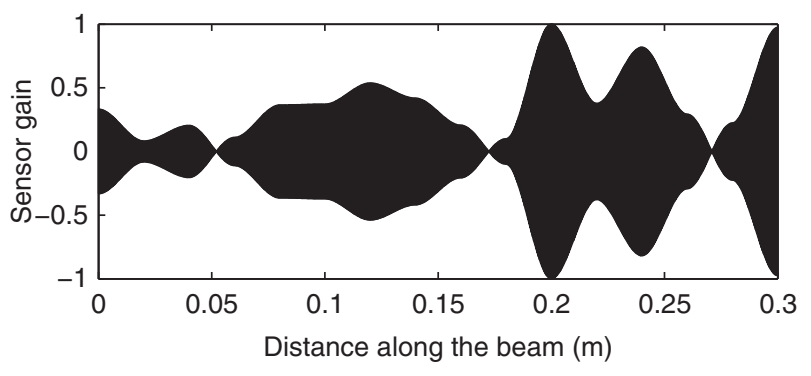

Figure 9. The sensor shape solely based on the robustness criterion for case 2, but using a threshold of $10^{-6}$ on the singular values in the solution.

The Pareto curve for case 2 is shown in Figure 6 and the sensor shape $t$ the corner of the Pareto curve is shown in Figure 7. The interpretation of the curves is similar to case 1 , except now the sensor shapes are more complex.

The sensor shapes shown in Figures 5 and 7 for the case $\mu=0$ are very complex. These shapes are based solely on the uncertainty criteria and are sensitive to the discretization because of the ill-conditioning in solving Equation (22). These ill-conditioning problems can be reduced by solving the set of equations using the singular value decomposition and only retaining those singular values above a given threshold, usually given as a factor of the highest singular value. Only the larger singular values have a significant influence on the value of the robustness penalty function $J_{r}$. In contrast the smaller singular values have the largest influence on the solution of the equations and hence on the shape of the sensor. This means that large differences in sensor shape only produce minor changes in the robustness penalty function and motivates neglecting the lower singular values. Figures 8 and 9 show the resulting sensor shape for cases 1 and 2, respectively when singular values lower than a threshold factor of $10^{-6}$ are neglected.

\section{CONCLUSIONS}

Uncertainties in the system need to be taken into account for the robust design of sensors and actuators 
for engineering dynamical systems. This article has considered the problem of designing modal sensors using a discrete approximation to the equations of motion for linear stochastic systems. Transducer shapes are represented by utilizing the underlying finite element shape functions. This allows the sensors to be designed by using a discrete approximation and the shape recovered by using the shape functions. Optimal shape design has been coupled with the stochastic finite element method to consider parametric uncertainty described using random fields. The sensor design problem only depends on the mode shapes of the structures, and so only the eigenvector statistics are needed to obtain the optimal shape for an uncertain system. A first-order perturbation based approach was proposed to obtain the secondorder covariance of the modal matrix in closed form. The analytical and numerical results for beam structures showed that the shape of the sensors of the stochastic system differs significantly from the corresponding deterministic system. However, sensors with shapes designed using a smoothness criterion perform very well for the linear beam structures with uncertainty considered in this article. Work is currently underway to extend the results to complex systems.

\section{ACKNOWLEDGMENTS}

S. Adhikari gratefully acknowledges the support of UK Engineering and Physical Sciences Research Council (EPSRC) through the award of an Advanced Research Fellowship and The Leverhulme Trust for the award of the Philip Leverhulme Prize.

\section{APPENDIX: STOCHASTIC PARTS OF THE ELEMENT MASS AND STIFFNESS MATRICES}

This appendix derives closed-form expressions for the random part of the element stiffness and mass matrices.

\section{Stochastic Element Stiffness Matrix}

Using the expression for $\varphi_{K j}$ from Equation (34) for odd $j$, the matrix $\mathbf{K}_{e_{j}}$ can be expressed from Equation (45) as:

$$
\begin{aligned}
\mathbf{K}_{e_{j}}= & E I_{0} \int_{0}^{\ell_{e}} \varphi_{\mathrm{K} j}\left(x_{e}+x\right) \mathbf{N}^{\prime \prime}(x) \mathbf{N}^{\prime \prime T}(x) \mathrm{d} x, \\
= & \frac{E I_{0}}{\sqrt{a+\sin \left(2 \omega_{j} a\right) / 2 \omega_{j}}} \boldsymbol{\Gamma} \\
& \times\left[\int_{0}^{\ell_{e}} \cos \left(\omega_{j}\left(x_{e}+x\right)\right) \mathbf{s}^{\prime \prime}(x) \mathbf{s}^{\prime \prime T}(x) \mathrm{d} x\right] \mathbf{\Gamma}^{T}, \\
= & \frac{4 E I_{0}}{\omega_{j}^{3} \ell_{e}^{6} \sqrt{a+\sin \left(2 \omega_{j} a\right) / 2 \omega_{j}}} \widetilde{\mathbf{K}}_{e_{j}} .
\end{aligned}
$$

Here $a=L / 2$ where $L$ is the length of the whole beam and:

$$
\begin{aligned}
\widetilde{\mathbf{K}}_{e_{j}}= & \frac{\omega_{j}^{3} \ell_{e}{ }^{6}}{4} \boldsymbol{\Gamma}\left[\int_{0}^{\ell_{e}} \cos \left(\omega_{j}\left(x_{e}+x\right)\right) \mathbf{s}^{\prime \prime}(x) \mathbf{s}^{\prime \prime T}(x) \mathrm{d} x\right] \boldsymbol{\Gamma}^{T} \\
= & \frac{\omega_{j}^{3} \ell_{e}{ }^{6}}{4} \boldsymbol{\Gamma} \int_{0}^{\ell_{e}} \cos \left(\omega_{j}\left(x_{e}+x\right)\right) \\
& \times\left[\begin{array}{cccc}
0 & 0 & 0 & 0 \\
0 & 0 & 0 & 0 \\
0 & 0 & 4 & 12 x \\
0 & 0 & 12 x & 36 x^{2}
\end{array}\right] \mathrm{d} x \boldsymbol{\Gamma}^{T} .
\end{aligned}
$$

The elements of the symmetric matrix $\widetilde{K}_{e_{j}}$ can be expressed as:

$$
\widetilde{\mathbf{K}}_{e_{j}}=\left[\begin{array}{cccc}
\widehat{K}_{e j_{11}} & \widehat{K}_{e j_{12}} & -\widehat{K}_{e j_{11}} & \widehat{K}_{e j_{12}} \\
& \widehat{K}_{e j_{22}} & -\widehat{K}_{e j_{12}} & \widehat{K}_{e j_{24}} \\
& & \widehat{K}_{e j_{11}} & -\widehat{K}_{e j_{12}} \\
& & & \widehat{K}_{e j_{22}}
\end{array}\right] .
$$

Performing the integrals appearing in Equation (70), the expressions of the four independent terms appearing in Equation (71) are:

$$
\begin{aligned}
\widehat{K}_{e j_{11}}= & -9 \omega_{j}^{2} \ell_{e}^{2} \sin \left(\omega_{j} x_{e}\right)+9 \sin \left(\omega_{j} \ell_{e}+\omega_{j} x_{e}\right) \omega_{j}^{2} \ell_{e}^{2} \\
& +36 \omega_{j} \ell_{e} \cos \left(\omega_{j} x_{e}\right)+36 \cos \left(\omega_{j} \ell_{e}+\omega_{j} x_{e}\right) \omega_{j} \ell_{e} \\
& +72 \sin \left(\omega_{j} x_{e}\right)-72 \sin \left(\omega_{j} \ell_{e}+\omega_{j} x_{e}\right),
\end{aligned}
$$

$$
\begin{aligned}
\widehat{K}_{e j_{12}}= & -3 \ell_{e}\left(2 \omega_{j}^{2} \ell_{e}^{2} \sin \left(\omega_{j} x_{e}\right)-\sin \left(\omega_{j} \ell_{e}+\omega_{j} x_{e}\right) \omega_{j}^{2} \ell_{e}^{2}\right. \\
& -7 \omega_{j} \ell_{e} \cos \left(\omega_{j} x_{e}\right)-5 \cos \left(\omega_{j} \ell_{e}+\omega_{j} x_{e}\right) \omega_{j} \ell_{e} \\
& \left.-12 \sin \left(\omega_{j} x_{e}\right)+12 \sin \left(\omega_{j} \ell_{e}+\omega_{j} x_{e}\right)\right),
\end{aligned}
$$

$$
\begin{aligned}
\widehat{K}_{e j_{22}}= & -\ell_{e}^{2}\left(4 \omega_{j}^{2} \ell_{e}^{2} \sin \left(\omega_{j} x_{e}\right)-\sin \left(\omega_{j} \ell_{e}+\omega_{j} x_{e}\right) \omega_{j}^{2} \ell_{e}^{2}\right. \\
& -12 \omega_{j} \ell_{e} \cos \left(\omega_{j} x_{e}\right)-6 \cos \left(\omega_{j} \ell_{e}+\omega_{j} x_{e}\right) \omega_{j} \ell_{e} \\
& \left.-18 \sin \left(\omega_{j} x_{e}\right)+18 \sin \left(\omega_{j} \ell_{e}+\omega_{j} x_{e}\right)\right),
\end{aligned}
$$

$$
\begin{aligned}
\widehat{K}_{e j_{24}}= & 3 \ell_{e}\left(2 \omega_{j}^{2} \ell_{e}^{2} \sin \left(\omega_{j} x_{e}\right)-\sin \left(\omega_{j} \ell_{e}+\omega_{j} x_{e}\right) \omega_{j}^{2} \ell_{e}^{2}\right. \\
& -7 \omega_{j} \ell_{e} \cos \left(\omega_{j} x_{e}\right)-5 \cos \left(\omega_{j} \ell_{e}+\omega_{j} x_{e}\right) \omega_{j} \ell_{e} \\
& \left.-12 \sin \left(\omega_{j} x_{e}\right)+12 \sin \left(\omega_{j} \ell_{e}+\omega_{j} x_{e}\right)\right) .
\end{aligned}
$$

The term associated with the even elements of the spectral expansion of the element stiffness matrix 
( $j$ even) can be obtained from Equation (45) as:

$$
\begin{aligned}
\mathbf{K}_{e_{j}}= & E I_{0} \int_{0}^{\ell_{e}} \varphi_{\mathrm{K} j}\left(x_{e}+x\right) \mathbf{N}^{\prime \prime}(x) \mathbf{N}^{\prime \prime T}(x) \mathrm{d} x, \\
= & \frac{E I_{0}}{\sqrt{a-\sin \left(2 \omega_{j} a\right) / 2 \omega_{j}}} \\
& \times \Gamma\left[\int_{0}^{\ell_{e}} \sin \left(\omega_{j}\left(x_{e}+x\right)\right) \mathbf{s}^{\prime \prime}(x) \mathbf{s}^{\prime \prime T}(x) \mathrm{d} x\right] \mathbf{\Gamma}^{T}, \\
= & \frac{4 E I_{0}}{\omega_{j}^{3} \ell_{e}^{6} \sqrt{a-\sin \left(2 \omega_{j} a\right) / 2 \omega_{j}}} \widetilde{\mathbf{K}}_{e_{j}},
\end{aligned}
$$

where

$$
\widetilde{\mathbf{K}}_{e_{j}}=\frac{\omega_{j}^{3} \ell_{e}^{6}}{4} \boldsymbol{\Gamma}\left[\int_{0}^{\ell_{e}} \sin \left(\omega_{j}\left(x_{e}+x\right)\right) \mathbf{s}^{\prime \prime}(x) \mathbf{s}^{\prime \prime T}(x) \mathrm{d} x\right] \boldsymbol{\Gamma}^{T} .
$$

The elements of the symmetric matrix $\widetilde{\mathbf{K}}_{e_{j}}$ can be expressed as:

$$
\widetilde{\mathbf{K}}_{e_{j}}=\left[\begin{array}{cccc}
\widehat{K}_{e j_{11}} & \widehat{K}_{e j_{12}} & -\widehat{K}_{e j_{11}} & \widehat{K}_{e j_{12}} \\
& \widehat{K}_{e j_{22}} & -\widehat{K}_{e j_{12}} & \widehat{K}_{e j_{24}} \\
& & \widehat{K}_{e j_{11}} & -\widehat{K}_{e j_{12}} \\
& & & \widehat{K}_{e j_{22}}
\end{array}\right] .
$$

Performing the integrals appearing in Equation (79), the expressions of the four independent terms appearing in the preceding equation can be obtained as:

$$
\begin{aligned}
\widehat{K}_{e_{11}}= & 9 \omega_{j}^{2} \ell_{e}^{2} \cos \left(\omega_{j} x_{e}\right)-9 \cos \left(\omega_{j} \ell_{e}+\omega_{j} x_{e}\right) \omega_{j}^{2} \ell_{e}^{2} \\
& +36 \omega_{j} \ell_{e} \sin \left(\omega_{j} x_{e}\right) \\
& +36 \sin \left(\omega_{j} \ell_{e}+\omega_{j} x_{e}\right) \omega_{j} \ell_{e} \\
& -72 \cos \left(\omega_{j} x_{e}\right)+72 \cos \left(\omega_{j} \ell_{e}+\omega_{j} x_{e}\right), \\
\widehat{K}_{e_{12}}= & 3 \ell_{e}\left(2 \omega_{j}^{2} \ell_{e}^{2} \cos \left(\omega_{j} x_{e}\right)-\cos \left(\omega_{j} \ell_{e}+\omega_{j} x_{e}\right) \omega_{j}^{2} \ell_{e}^{2}\right. \\
& +7 \omega_{j} \ell_{e} \sin \left(\omega_{j} x_{e}\right)+5 \sin \left(\omega_{j} \ell_{e}+\omega_{j} x_{e}\right) \omega_{j} \ell_{e} \\
& \left.-12 \cos \left(\omega_{j} x_{e}\right)+12 \cos \left(\omega_{j} \ell_{e}+\omega_{j} x_{e}\right)\right), \\
\widehat{K}_{e_{22}}= & \ell_{e}^{2}\left(4 \omega_{j}^{2} \ell_{e}^{2} \cos \left(\omega_{j} x_{e}\right)-\cos \left(\omega_{j} \ell_{e}+\omega_{j} x_{e}\right) \omega_{j}^{2} \ell_{e}^{2}\right. \\
& +12 \omega_{j} \ell_{e} \sin \left(\omega_{j} x_{e}\right)+6 \sin \left(\omega_{j} \ell_{e}+\omega_{j} x_{e}\right) \omega_{j} \ell_{e} \\
& \left.-18 \cos \left(\omega_{j} x_{e}\right)+18 \cos \left(\omega_{j} \ell_{e}+\omega_{j} x_{e}\right)\right), \\
\widehat{K}_{e_{24}}= & -3 \ell_{e}\left(2 \omega_{j}^{2} \ell_{e}^{2} \cos \left(\omega_{j} x_{e}\right)-\cos \left(\omega_{j} \ell_{e}+\omega_{j} x_{e}\right) \omega_{j}^{2} \ell_{e}^{2}\right. \\
& +7 \omega_{j} \ell_{e} \sin \left(\omega_{j} x_{e}\right)+5 \sin \left(\omega_{j} \ell_{e}+\omega_{j} x_{e}\right) \omega_{j} \ell_{e} \\
& \left.-12 \cos \left(\omega_{j} x_{e}\right)+12 \cos \left(\omega_{j} \ell_{e}+\omega_{j} x_{e}\right)\right) .
\end{aligned}
$$

\section{Stochastic Element Mass Matrix}

Using the expression of $\varphi_{\mathbf{M} j}$ from Equation (34) for odd $j$, the matrix $\mathbf{M}_{e_{j}}$ can be expressed from Equation (45) as:

$$
\begin{aligned}
\mathbf{M}_{e_{j}}= & m_{0} \int_{0}^{\ell_{e}} \varphi_{\mathrm{M} j}\left(x_{e}+x\right) \mathbf{N}(x)^{T}(x) \mathrm{d} x, \\
= & \frac{m_{0}}{\sqrt{a+\sin \left(2 \omega_{j} a\right) / 2 \omega_{j}}} \\
& \times \boldsymbol{\Gamma}\left[\int_{0}^{\ell_{e}} \cos \left(\omega_{j}\left(x_{e}+x\right)\right) \mathbf{s}(x) \mathbf{s}^{T}(x) \mathrm{d} x\right] \mathbf{\Gamma}^{T}, \\
= & \frac{m_{0}}{\omega_{j}^{7} \ell_{e} \sqrt{a+\sin \left(2 \omega_{j} a\right) / 2 \omega_{j}}} \tilde{\mathbf{M}}_{e_{j}},
\end{aligned}
$$

where

$$
\begin{aligned}
\tilde{\mathbf{M}}_{e_{j}}= & \left(\omega_{j}^{7} \ell_{e}^{6}\right) \boldsymbol{\Gamma}\left[\int_{0}^{\ell_{e}} \cos \left(\omega_{j}\left(x_{e}+x\right)\right) \mathbf{s}(x) \mathbf{s}^{T}(x) \mathrm{d} x\right] \boldsymbol{\Gamma}^{T} \\
= & \left(\omega_{j}^{7} \ell_{e}^{6}\right) \boldsymbol{\Gamma} \int_{0}^{\ell_{e}} \cos \left(\omega_{j}\left(x_{e}+x\right)\right) \\
& \times\left[\begin{array}{cccc}
1 & x & x^{2} & x^{3} \\
x & x^{2} & x^{3} & x^{4} \\
x^{2} & x^{3} & x^{4} & x^{5} \\
x^{3} & x^{4} & x^{5} & x^{6}
\end{array}\right] \mathrm{d} x \boldsymbol{\Gamma}^{T}
\end{aligned}
$$

The elements of the symmetric matrix $\tilde{\mathbf{M}}_{e_{j}}$ can be expressed as:

$$
\tilde{\mathbf{M}}_{e_{j}}=\left[\begin{array}{cccc}
\widehat{M}_{e j_{11}} & \widehat{M}_{e j_{12}} & -\widehat{M}_{e j_{13}} & \widehat{M}_{e j_{14}} \\
& \widehat{M}_{e j_{22} 2} & -\widehat{M}_{e j_{14}} & \widehat{M}_{e j_{24}} \\
& & \widehat{M}_{e j_{11}} & -\widehat{M}_{e j_{12}} \\
& & & \widehat{M}_{e j_{22}}
\end{array}\right] .
$$

Performing the integrals appearing in Equation (88), the expressions of the six independent terms appearing in the preceding equation can be obtained as:

$$
\begin{aligned}
\widehat{M}_{e j_{11}}= & 24 \omega_{j}^{3} \ell_{e}^{3} \cos \left(\omega_{j} x_{e}\right)+1440 \cos \left(\omega_{j} \ell_{e}+\omega_{j} x_{e}\right) \omega_{j} \ell_{e} \\
& -216 \omega_{j}^{2} \ell_{e}^{2} \sin \left(\omega_{j} x_{e}\right)+1440 \omega_{j} \ell_{e} \cos \left(\omega_{j} x_{e}\right) \\
& +216 \sin \left(\omega_{j} \ell_{e}+\omega_{j} x_{e}\right) \omega_{j}^{2} \ell_{e}^{2} \\
& -12 \omega_{j}^{4} \ell_{e}^{4} \sin \left(\omega_{j} x_{e}\right)-\ell_{e}^{6} \omega_{j}^{6} \sin \left(\omega_{j} x_{e}\right) \\
& -2880 \sin \left(\omega_{j} \ell_{e}+\omega_{j} x_{e}\right)+2880 \sin \left(\omega_{j} x_{e}\right)
\end{aligned}
$$




$$
\begin{aligned}
\widehat{M}_{e j_{12}}= & -\ell_{e}\left(12 \omega_{j}^{3} \ell_{e}^{3} \cos \left(\omega_{j} x_{e}\right)-600 \cos \left(\omega_{j} \ell_{e}+\omega_{j} x_{e}\right) \omega_{j} \ell_{e}\right. \\
& +192 \omega_{j}^{2} \ell_{e}{ }^{2} \sin \left(\omega_{j} x_{e}\right)-840 \omega_{j} \ell_{e} \cos \left(\omega_{j} x_{e}\right) \\
& -72 \sin \left(\omega_{j} \ell_{e}+\omega_{j} x_{e}\right) \omega_{j}^{2} \ell_{e}^{2}+4 \omega_{j}^{4} \ell_{e}^{4} \sin \left(\omega_{j} x_{e}\right) \\
& +1440 \sin \left(\omega_{j} \ell_{e}+\omega_{j} x_{e}\right)-1440 \sin \left(\omega_{j} x_{e}\right) \\
& \left.+\ell_{e}^{5} \omega_{j}^{5} \cos \left(\omega_{j} x_{e}\right)\right) \\
\widehat{M}_{e j_{13}}= & -12 \cos \left(\omega_{j} \ell_{e}+\omega_{j} x_{e}\right) \omega_{j}^{3} \ell_{e}^{3} \\
& -6 \sin \left(\omega_{j} \ell_{e}+\omega_{j} x_{e}\right) \omega_{j}^{4} \ell_{e}{ }^{4} \\
& -12 \omega_{j}^{3} \ell_{e}^{3} \cos \left(\omega_{j} x_{e}\right)-1440 \cos \left(\omega_{j} \ell_{e}+\omega_{j} x_{e}\right) \omega_{j} \ell_{e} \\
& +216 \omega_{j}^{2} \ell_{e}{ }^{2} \sin \left(\omega_{j} x_{e}\right)-1440 \omega_{j} \ell_{e} \cos \left(\omega_{j} x_{e}\right) \\
& -216 \sin \left(\omega_{j} \ell_{e}+\omega_{j} x_{e}\right) \omega_{j}^{2} \ell_{e}{ }^{2}+6 \omega_{j}^{4} \ell_{e}^{4} \sin \left(\omega_{j} x_{e}\right) \\
& +2880 \sin \left(\omega_{j} \ell_{e}+\omega_{j} x_{e}\right)-2880 \sin \left(\omega_{j} x_{e}\right),
\end{aligned}
$$

$$
\begin{aligned}
\widehat{M}_{e j_{14}}= & -2 \ell_{e}\left(\omega_{j}^{4} \ell_{e}^{4} \sin \left(\omega_{j} x_{e}\right)-3 \omega_{j}^{3} \ell_{e}^{3} \cos \left(\omega_{j} x_{e}\right)\right. \\
& +9 \cos \left(\omega_{j} \ell_{e}+\omega_{j} x_{e}\right) \omega_{j}^{3} \ell_{e}^{3}+36 \omega_{j}^{2} \ell_{e}^{2} \sin \left(\omega_{j} x_{e}\right) \\
& -96 \sin \left(\omega_{j} \ell_{e}+\omega_{j} x_{e}\right) \omega_{j}^{2} \ell_{e}^{2}-300 \omega_{j} \ell_{e} \cos \left(\omega_{j} x_{e}\right) \\
& -420 \cos \left(\omega_{j} \ell_{e}+\omega_{j} x_{e}\right) \omega_{j} \ell_{e}-720 \sin \left(\omega_{j} x_{e}\right) \\
& \left.+720 \sin \left(\omega_{j} \ell_{e}+\omega_{j} x_{e}\right)\right)
\end{aligned}
$$

$$
\begin{aligned}
\widehat{M}_{e j_{22}}= & 2 \ell_{e}^{2}\left(12 \sin \left(\omega_{j} \ell_{e}+\omega_{j} x_{e}\right) \omega_{j}^{2} \ell_{e}^{2}+240 \omega_{j} \ell_{e} \cos \left(\omega_{j} x_{e}\right)\right. \\
& -72 \omega_{j}^{2} \ell_{e}^{2} \sin \left(\omega_{j} x_{e}\right)+120 \cos \left(\omega_{j} \ell_{e}+\omega_{j} x_{e}\right) \omega_{j} \ell_{e} \\
& +360 \sin \left(\omega_{j} x_{e}\right)-360 \sin \left(\omega_{j} \ell_{e}+\omega_{j} x_{e}\right) \\
& \left.+\omega_{j}^{4} \ell_{e}^{4} \sin \left(\omega_{j} x_{e}\right)-12 \omega_{j}^{3} \ell_{e}^{3} \cos \left(\omega_{j} x_{e}\right)\right),
\end{aligned}
$$

$$
\begin{aligned}
\widehat{M}_{e j_{24}}= & -6 \ell_{e}^{2}\left(\omega_{j}^{3} \ell_{e}^{3} \cos \left(\omega_{j} x_{e}\right)+\cos \left(\omega_{j} \ell_{e}+\omega_{j} x_{e}\right) \omega_{j}^{3} \ell_{e}{ }^{3}\right. \\
& +12 \omega_{j}^{2} \ell_{e}^{2} \sin \left(\omega_{j} x_{e}\right)-12 \sin \left(\omega_{j} \ell_{e}+\omega_{j} x_{e}\right) \omega_{j}^{2} \ell_{e}{ }^{2} \\
& -60 \omega_{j} \ell_{e} \cos \left(\omega_{j} x_{e}\right)-60 \cos \left(\omega_{j} \ell_{e}+\omega_{j} x_{e}\right) \omega_{j} \ell_{e} \\
& \left.-120 \sin \left(\omega_{j} x_{e}\right)+120 \sin \left(\omega_{j} \ell_{e}+\omega_{j} x_{e}\right)\right) .
\end{aligned}
$$

The term associated with the even elements of the spectral expansion of the element mass matrix can be obtained from Equation (45) as:

$$
\begin{aligned}
\mathbf{M}_{e_{j}}= & m_{0} \int_{0}^{\ell_{e}} \varphi_{\mathrm{M} j}\left(x_{e}+x\right) \mathbf{N}(x) \mathbf{N}^{T}(x) \mathrm{d} x, \\
= & \frac{m_{0}}{\sqrt{a-\sin \left(2 \omega_{j} a\right) / 2 \omega_{j}}} \\
& \times \boldsymbol{\Gamma}\left[\int_{0}^{\ell_{e}} \sin \left(\omega_{j}\left(x_{e}+x\right)\right) \mathrm{s}(x) \mathrm{s}^{T}(x) \mathrm{d} x\right] \boldsymbol{\Gamma}^{T}, \\
= & \frac{m_{0}}{\omega_{j}^{7} \ell_{e}{ }^{6} \sqrt{a-\sin \left(2 \omega_{j} a\right) / 2 \omega_{j}}} \tilde{\mathbf{M}}_{e_{j}},
\end{aligned}
$$

where

$$
\tilde{\mathbf{M}}_{e_{j}}=\left(\omega_{j}^{7} \ell_{e}^{6}\right) \boldsymbol{\Gamma}\left[\int_{0}^{\ell_{e}} \sin \left(\omega_{j}\left(x_{e}+x\right)\right) \mathbf{s}(x) \mathbf{s}^{T}(x) \mathrm{d} x\right] \boldsymbol{\Gamma}^{T}
$$

The elements of the symmetric matrix $\tilde{\mathbf{M}}_{e_{j}}$ can be expressed as:

$$
\tilde{\mathbf{M}}_{e_{j}}=\left[\begin{array}{cccc}
\widehat{M}_{e j_{11}} & \widehat{M}_{e j_{12}} & -\widehat{M}_{e j_{13}} & \widehat{M}_{e j_{14}} \\
& \widehat{M}_{e j_{22}} & -\widehat{M}_{e j_{14}} & \widehat{M}_{e_{j_{24}}} \\
& & \widehat{M}_{e j_{11}} & -\widehat{M}_{e j_{12}} \\
& & & \widehat{M}_{e j_{22}}
\end{array}\right] .
$$

Performing the integrals appearing in Equation (99), the expressions of the six independent terms appearing in the preceding equation can be obtained as:

$$
\begin{aligned}
\widehat{M}_{e_{11}=} & -216 \cos \left(\omega_{j} \ell_{e}+\omega_{j} x_{e}\right) \omega_{j}^{2} \ell_{e}{ }^{2} \\
& +1440 \sin \left(\omega_{j} \ell_{e}+\omega_{j} x_{e}\right) \omega_{j} \ell_{e}+\omega_{j}^{6} \ell_{e}{ }^{6} \cos \left(\omega_{j} x_{e}\right) \\
& +12 \ell_{e}{ }^{4} \omega_{j}^{4} \cos \left(\omega_{j} x_{e}\right)+216 \omega_{j}{ }^{2} \ell_{e}{ }^{2} \cos \left(\omega_{j} x_{e}\right) \\
& +1440 \omega_{j} \ell_{e} \sin \left(\omega_{j} x_{e}\right)+24 \ell_{e}^{3} \omega_{j}^{3} \sin \left(\omega_{j} x_{e}\right) \\
& -2880 \cos \left(\omega_{j} x_{e}\right)+2880 \cos \left(\omega_{j} \ell_{e}+\omega_{j} x_{e}\right),
\end{aligned}
$$

$$
\begin{aligned}
\widehat{M}_{e_{12}}= & -\ell_{e}\left(72 \cos \left(\omega_{j} \ell_{e}+\omega_{j} x_{e}\right) \omega_{j}^{2} \ell_{e}^{2}\right. \\
& -600 \sin \left(\omega_{j} \ell_{e}+\omega_{j} x_{e}\right) \omega_{j} \ell_{e}-4 \ell_{e}^{4} \omega_{j}^{4} \cos \left(\omega_{j} x_{e}\right) \\
& -192 \omega_{j}^{2} \ell_{e}^{2} \cos \left(\omega_{j} x_{e}\right)-840 \omega_{j} \ell_{e} \sin \left(\omega_{j} x_{e}\right) \\
& +12 \ell_{e}^{3} \omega_{j}^{3} \sin \left(\omega_{j} x_{e}\right)+1440 \cos \left(\omega_{j} x_{e}\right) \\
& \left.-1440 \cos \left(\omega_{j} \ell_{e}+\omega_{j} x_{e}\right)+\omega_{j}^{5} \ell_{e}^{5} \sin \left(\omega_{j} x_{e}\right)\right),
\end{aligned}
$$

$$
\begin{aligned}
\widehat{M}_{e_{13}}= & 216 \cos \left(\omega_{j} \ell_{e}+\omega_{j} x_{e}\right) \omega_{j}^{2} \ell_{e}^{2} \\
& -1440 \sin \left(\omega_{j} \ell_{e}+\omega_{j} x_{e}\right) \omega_{j} \ell_{e} \\
& -12 \sin \left(\omega_{j} \ell_{e}+\omega_{j} x_{e}\right) \omega_{j}^{3} \ell_{e}^{3}-6 \ell_{e}{ }^{4} \omega_{j}^{4} \cos \left(\omega_{j} x_{e}\right) \\
& -216 \omega_{j}^{2} \ell_{e}^{2} \cos \left(\omega_{j} x_{e}\right)-1440 \omega_{j} \ell_{e} \sin \left(\omega_{j} x_{e}\right) \\
& +6 \cos \left(\omega_{j} \ell_{e}+\omega_{j} x_{e}\right) \omega_{j}^{4} \ell_{e}{ }^{4}-12 \ell_{e}{ }^{3} \omega_{j}^{3} \sin \left(\omega_{j} x_{e}\right) \\
& +2880 \cos \left(\omega_{j} x_{e}\right)-2880 \cos \left(\omega_{j} \ell_{e}+\omega_{j} x_{e}\right),
\end{aligned}
$$

$$
\begin{aligned}
\widehat{M}_{e_{14}}= & 2 \ell_{e}\left(\ell_{e}^{4} \omega_{j}^{4} \cos \left(\omega_{j} x_{e}\right)+3 \ell_{e}^{3} \omega_{j}^{3} \sin \left(\omega_{j} x_{e}\right)\right. \\
& -9 \sin \left(\omega_{j} \ell_{e}+\omega_{j} x_{e}\right) \omega_{j}^{3} \ell_{e}^{3}+36 \omega_{j}^{2} \ell_{e}^{2} \cos \left(\omega_{j} x_{e}\right) \\
& -96 \cos \left(\omega_{j} \ell_{e}+\omega_{j} x_{e}\right) \omega_{j}^{2} \ell_{e}^{2}+300 \omega_{j} \ell_{e} \sin \left(\omega_{j} x_{e}\right) \\
& +420 \sin \left(\omega_{j} \ell_{e}+\omega_{j} x_{e}\right) \omega_{j} \ell_{e}-720 \cos \left(\omega_{j} x_{e}\right) \\
& \left.+720 \cos \left(\omega_{j} \ell_{e}+\omega_{j} x_{e}\right)\right)
\end{aligned}
$$




$$
\begin{aligned}
\widehat{M}_{e_{22}}= & -2 \ell_{e}^{2}\left(12 \cos \left(\omega_{j} \ell_{e}+\omega_{j} x_{e}\right) \omega_{j}^{2} \ell_{e}^{2}\right. \\
& -120 \sin \left(\omega_{j} \ell_{e}+\omega_{j} x_{e}\right) \omega_{j} \ell_{e}-72 \omega_{j}^{2} \ell_{e}^{2} \cos \left(\omega_{j} x_{e}\right) \\
& -240 \omega_{j} \ell_{e} \sin \left(\omega_{j} x_{e}\right)+12 \ell_{e}^{3} \omega_{j}^{3} \sin \left(\omega_{j} x_{e}\right) \\
& +360 \cos \left(\omega_{j} x_{e}\right)-360 \cos \left(\omega_{j} \ell_{e}+\omega_{j} x_{e}\right) \\
& \left.+\ell_{e}^{4} \omega_{j}^{4} \cos \left(\omega_{j} x_{e}\right)\right), \\
\widehat{M}_{e_{24}}= & -6 \ell_{e}{ }^{2}\left(\ell_{e}^{3} \omega_{j}^{3} \sin \left(\omega_{j} x_{e}\right)+\sin \left(\omega_{j} \ell_{e}+\omega_{j} x_{e}\right) \omega_{j}^{3} \ell_{e}^{3}\right. \\
& -12 \omega_{j}^{2} \ell_{e}^{2} \cos \left(\omega_{j} x_{e}\right)+12 \cos \left(\omega_{j} \ell_{e}+\omega_{j} x_{e}\right) \omega_{j}^{2} \ell_{e}{ }^{2} \\
& -60 \omega_{j} \ell_{e} \sin \left(\omega_{j} x_{e}\right)-60 \sin \left(\omega_{j} \ell_{e}+\omega_{j} x_{e}\right) \omega_{j} \ell_{e} \\
& \left.+120 \cos \left(\omega_{j} x_{e}\right)-120 \cos \left(\omega_{j} \ell_{e}+\omega_{j} x_{e}\right)\right) .
\end{aligned}
$$

\section{REFERENCES}

Adhikari, S. 1999. "Modal Analysis of Linear Asymmetric Non-conservative Systems," ASCE Journal of Engineering Mechanics, 125(12):1372-1379.

Adhikari, S. 2004a. "Complex Modes in Stochastic Systems," Advances in Vibration Engineering, 3(1):1-11.

Adhikari, S. 2004b. "Optimal Complex Modes and an Index of Damping Non-proportionality," Mechanical System and Signal Processing, 18(1):1-27.

Adhikari, S. 2006. "Random Eigenvalue Problems Revisited," Sädhanā - Proceedings of the Indian Academy of Engineering Sciences, 31(4):293-314. (Special Issue on Probabilistic Structural Dynamics and Earthquake Engineering).

Adhikari, S. 2007. "Joint Statistics of Natural Frequencies of Stochastic Dynamic Systems," Computational Mechanics, 40(4): 739-752.

Adhikari, S. and Friswell, M.I. 2007. "Random Matrix Eigenvalue Problems in Structural Dynamics," International Journal for Numerical Methods in Engineering, 69(3):562-591.

Ahmadian, H., Mottershead, J.E. and Friswell, M.I. 1998. "Regularisation Methods for Finite Element Model Updating," Mechanical Systems and Signal Processing, 12(1):47-64.

Benaroya, H. 1992. "Random Eigenvalues, Algebraic Methods and Structural Dynamic Models," Applied Mathematics and Computation, 52:37-66.

Boyce, W.E. 1968. "Random Eigenvalue Problems," Probabilistic Methods in Applied Mathematics, Academic Press, New York.

Collins, J.D. and Thomson, W.T. 1969. "The Eigenvalue Problem for Structural Systems with Statistical Properties," AIAA Journal, 7(4):642-648.

den Nieuwenhof, B.V. and Coyette, J.-P. 2003. "Modal Approaches for the Stochastic Finite Element Analysis of Structures with Material and Geometric Uncertainties," Computer Methods in Applied Mechanics and Engineering, 192(33-34):3705-3729.

Donoso, A. and Bellido, J.C. 2009a. "Distributed Piezoelectric Modal Sensors for Circular Plates," Journal of Sound and Vibration, 319(1-2):50-57.

Donoso, A. and Bellido, J.C. 2009b. "Tailoring Distributed Modal Sensors for In-plane Modal Filtering," Smart Materials and Structures, 18(3), paper no. 037002: 1-4.

Elka, A. and Bucher, I. 2009. "Optimal Electrode Shaping for Precise Modal Electromechanical Filtering," Structural and Multidisciplinary Optimization, 38(6):627-641.

Fox, R.L. and Kapoor, M.P. 1968. "Rates of Change of Eigenvalues and Eigenvectors," AIAA Journal, 6(12):2426-2429.

Friswell, M.I. 1999. "Partial and Segmented Modal Sensors for Beam Structures," Journal of Vibration and Control, 5:619-637.

Friswell, M.I. 2001. "On the Design of Modal Actuators and Sensors," Journal of Sound and Vibration, 241:361-372.
Gawronski, W. 2000. "Modal Actuators and Sensors," Journal of Sound and Vibration, 229:1013-1022.

Ghanem, R. and Spanos, P.D. 1991. Stochastic Finite Elements: A Spectral Approach, Springer-Verlag, New York, USA.

Ghosh, D., Ghanem, R.G. and Red-Horse, J. 2005. "Analysis of Eigenvalues and Modal Interaction of Stochastic Systems," AIAA Journal, 43(10):2196-2201.

Grigoriu, M. 1992. "A Solution of Random Eigenvalue Problem by Crossing Theory," Journal of Sound and Vibration, 158(1):69-80.

Hála, M. 1994. "Method of Ritz for Random Eigenvalue Problems," Kybernetika, 30(3):263-269.

Hansen, P.C. 1992. "Analysis of Discrete Ill-posed Problems by Means of the L-curve," SIAM Review, 34:561-580.

Hansen, P.C. 1994. "Regularisation Tools: A MATLAB Package for Analysis and Solution of Discrete Ill-posed Problems," Numerical Algorithms, 6:1-35.

Hansen, P.C. and O'Leary, D.P. 1993. "The Use of the L-curve in the Regularisation of Discrete Ill-posed Problems," SIAM Journal of Scientific Computation, 14:1487-1503.

Hart, G.C. 1973. "Eigenvalue Uncertainties in Stressed Structure," ASCE Journal of Engineering Mechanics, 99(EM3):481-494.

Hasselman, T.K. and Hart, G.C. 1972. "Modal Analysis of Random Structural System," ASCE Journal of Engineering Mechanics, 98(EM3):561-579.

Hsu, C.-Y., Lin, C.-C. and Gaul, L. 1998. "Vibration and Sound Radiation Controls of Beams Using Layered Modal Sensors and Actuators," Smart Materials and Structures, 7:446-455.

Huang, Y.T., Wu, W.J., Chen, Y.Y. and Lee, C.K. 2007a. "A Modal Actuator-based Single-layer Piezoelectric Transformer for Coilless Soft Switching Inverters," Smart Materials and Structures, 16(6):2046-2055.

Huang, Y.-T., Wu, W.-J., Wang, Y.-C. and Lee, C.-K. 2007b. "Multilayer Modal Actuator-based Piezoelectric Transformers," IEEE Transactions on Ultransonics Ferroelectrics and Frequency Control, 54(2):359-367.

Ibrahim, R.A. 1987. "Structural Dynamics with Parameter Uncertainties," Applied Mechanics Reviews, ASME, 40(3):309-328.

Jian, K. and Friswell, M.I. 2006. "Designing Distributed Modal Sensors for Plate Structures Using Finite Element Analysis," Mechanical Systems and Signal Processing, 20(8):2290-2304.

Kleiber, M. and Hien, T.D. 1992. The Stochastic Finite Element Method, John Wiley, Chichester.

Lee, C. and Singh, R. 1994. "Analysis of Discrete Vibratory Systems with Parameter Uncertainties, part I: Eigensolution," Journal of Sound and Vibration, 174(3):379-394.

Lee, C.-K. and Moon, F.C. 1990. "Modal Sensors/Actuators," Journal of Applied Mechanics, 57:434-441.

Manohar, C.S. and Adhikari, S. 1998a. "Dynamic Stiffness of Randomly Parametered Beams," Probabilistic Engineering Mechanics, 13(1):39-51.

Manohar, C.S. and Adhikari, S. 1998b. "Statistical Analysis of Vibration Energy Flow in Randomly Parametered Trusses," Journal of Sound and Vibration, 217(1):43-74.

Manohar, C.S. and Gupta, S. 2003. "Modeling and Evaluation of Structural Reliability: Current Status and Future Directions," In: Jagadish, K.S. and Iyengar, R.N. (eds), Research Reviews in Structural Engineering, Golden Jubilee Publications of Department of Civil Engineering, Indian Institute of Science, Bangalore, pp. 90-187, University Press, Hyderabad.

Manohar, C.S. and Ibrahim, R.A. 1999. "Progress in Structural Dynamics with Stochastic Parameter Variations: 1987 to 1998," Applied Mechanics Reviews, ASME, 52(5):177-197.

Mehlhose, S., vom Scheidt, J. and Wunderlich, R. 1999. "Random Eigenvalue Problems for Bending Vibrations of Beams," Zeitschrift Fur Angewandte Mathematik Und Mechanik, 79(10): 693-702.

Mukherjee, A. and Joshi, S. 2002. "Piezoelectric Sensor and Actuator Spatial Design for Shape Control of Piezolaminated Plates," AIAA Journal, 40(6):1204-1210. 
Nair, P.B. and Keane, A.J. 2003. "An Approximate Solution Scheme for the Algebraic Random Eigenvalue Problem," Journal of Sound and Vibration, 260(1):45-65.

Papoulis, A. and Pillai, S.U. 2002. Probability, Random Variables and Stochastic Processes, 4th edn, McGraw-Hill, Boston, USA.

Rahman, S. 2006. "A Solution of the Random Eigenvalue Problem by a Dimensional Decomposition Method," International Journal for Numerical Methods in Engineering, 67(9):1318-1340.

Ramu, S.A. and Ganesan, R. 1992a. "Stability Analysis of a Stochastic Column Subjected to Stochastically Distributed Loadings Using the Finite Element Method," Finite Elements in Analysis and Design, 11:105-115.

Ramu, S.A. and Ganesan, R. 1992b. "Stability of Stochastic Leipholz Column with Stochastic Loading," Archive of Applied Mechanics, 62:363-375.

Ramu, S.A. and Ganesan, R. 1993a. "A Galerkin Finite Element Technique for Stochastic Field Problems," Computer Methods in Applied Mechanics and Engineering, 105:315-331.
Ramu, S.A. and Ganesan, R. 1993b. "Parametric Instability of Stochastic Columns," International Journal of Solids and Structures, 30(10):1339-1354.

Sankar, T.S., Ramu, S.A. and Ganesan, R. 1993. "Stochastic Finite Element Analysis for High Speed Rotors," Transactions of ASME, Journal of Vibration and Acoustics, 115:59-64.

Scheidt, J.V. and Purkert, W. 1983. Random Eigenvalue Problems, North Holland, New York.

Song, D., Chen, S. and Qiu, Z. 1995. "Stochastic Sensitivity Analysis of Eigenvalues and Eigenvectors," Computer and Structures, 54(5):891-896.

Titurus, B. and Friswell, M.I. 2008. "Regularization in Model Updating," International Journal for Numerical Methods in Engineering, 75(4):440-478.

Verhoosel, C.V., Gutiérrez, M.A. and Hulshoff, S.J. 2006. "Iterative Solution of the Random Eigenvalue Problem with Application to Spectral Stochastic Finite Element Systems," International Journal for Numerical Methods in Engineering, 68(4):401-424. 